\title{
A Bayesian Nonparametric Approach to Discover Clinico-Genetic Associations across Cancer Types
}

\author{
Melanie F. Pradier ${ }^{1,2,3, \dagger}$, Stephanie L. Hyland ${ }^{1,4,5,6}$, Stefan G. Stark ${ }^{1,5,6}$, Kjong \\ Lehmann ${ }^{1,5,6,8}$, Julia E. Vogt ${ }^{5,7,8}$, Fernando Perez-Cruz ${ }^{2,9, *}$, and Gunnar Rätsch ${ }^{1,5,6,8,10, \dagger \cdot *}$ \\ ${ }^{1}$ Computational Biology Program, Memorial Sloan Kettering Cancer Center, New York, U.S.A. \\ ${ }^{2}$ University Carlos III in Madrid, Leganés, Spain \\ ${ }^{3}$ School of Engineering and Applied Sciences, Harvard University, Cambridge, USA \\ ${ }^{4}$ Ph.D. Program in Computational Biology and Medicine, Weill Cornell Medicine, New York, U.S.A. \\ ${ }^{5}$ Department of Computer Science, ETH Zürich, Zürich, Switzerland \\ ${ }^{6}$ Medical Informatics Group, University Hospital Zürich, Zürich, Switzerland \\ ${ }^{7}$ Department of Mathematics and Computer Science, University of Basel, Basel, Switzerland \\ ${ }^{8}$ Swiss Institute for Bioinformatics, Lausanne, Switzerland \\ ${ }^{9}$ Swiss Data Science Center, ETH Zürich and EPFL Lausanne, Switzerland \\ ${ }^{10}$ Department of Biology, ETH Zürich, Zürich, Switzerland \\ † Contact: melanie@seas.harvard.edu, gunnar.ratsch@ratschlab.org \\ ${ }^{*}$ These authors jointly directed this work
}

April 29, 2019

\begin{abstract}
Motivation: Personalized medicine aims at combining genetic, clinical, and environmental data to improve medical diagnosis and disease treatment, tailored to each patient. This paper presents a Bayesian nonparametric (BNP) approach to identify genetic associations with clinical/environmental features in cancer. We propose an unsupervised approach to generate data-driven hypotheses and bring potentially novel insights about cancer biology. Our model combines somatic mutation information at gene-level with features extracted from the Electronic Health Record. We propose a hierarchical approach, the hierarchical Poisson factor analysis (HPFA) model, to share information across patients having different types of cancer. To discover statistically significant associations, we combine Bayesian modeling with bootstrapping techniques and correct for multiple hypothesis testing.
\end{abstract}

Results: Using our approach, we empirically demonstrate that we can recover well-known associations in cancer literature. We compare the results of H-PFA with two other classical methods in the field: case-control (CC) setups, and linear mixed models (LMMs).

\section{Introduction}

Cancer encompasses not one, but a large group of genetic diseases involving abnormal cell growth with the potential to invade or spread to other parts of the body. Although a small set of universal underlying principles were identified, the so-called "hallmarks" of cancer [19, 20], each type of cancer presents unique properties, making this disease very hard to treat [37, 22, 43]. 
Genetic association studies have been successful in relating somatic mutation to carcinogenesis, but has been limited to the detection of common large-effect variants in the presence of only small cohorts $[9,27,8]$. Finding somatic driver mutations is even more challenging since these mutations are often rare. The large phenotypic heterogeneity, which reduces statistical power in the discovery method, causes some associations to remain hidden [38, 29, 40]. Cohort sizes tend to be small, especially in rare cancers, which makes the discovery of small effect size associations difficult [3]. Additionally, carcinogenesis is driven by the accumulation of mutations that may act epistatically or pleiotropically during the disease, further reducing the power of typical approaches $[48,11,42,12]$. To overcome these difficulties, new approaches for interpreting genetic variation across different cancer types are required.

In recent years, efforts to mine electronic health records (EHRs) show promise to impact nearly every aspect of healthcare [26]. The adoption of EHRs in hospitals has increased dramatically and has become a powerful resource for phenotyping $[2,32]$, with the potential for establishing new patient-stratification principles and for revealing unknown disease correlations. Integrating EHRs data with genetic data will also give a better understanding of genotypephenotype relationships [49]. EHRs consist of both structured and unstructured information. Structured data is a valuable source of information that includes billing codes, laboratory reports, physiological measurements, and demographic information, among others. Yet, most of the clinical data comes as unstructured notes, e.g., around 98\% of the EHRs [26]. These include a broad spectrum of clinically-relevant information which might be useful to identify novel phenotypic relationships so far unknown by the clinicians $[32,26]$.

This work presents a joint generative model to discover associations between somatic mutations and clinical features in cancer that deals with phenotype heterogeneity, small cohort size, epistasis and pleiotropy in a straightforward way. Our method infers latent topics from the clinical text and genetic information, capturing complex interactions between groups of genes and clinical features. It is directly inspired by the Poisson factorization model for recommendation systems [16], with three important differences.

First, we introduce confounding effects as conditional variables, i.e., variables that might cause spurious associations to appear. In particular, our model considers multiple types of cancer together (the type of cancer is treated as a confounder) and shares information among all patients in a hierarchical fashion. Indeed, most cancers are known to share common pathogenesis despite specificities of the cell type and tissue origin [43]. By doing so, specific effects for each type of cancer can be isolated, and additional (less well-known) associations with somatic mutations of smaller effect size can be obtained.

Second, we force sparsity on the textual and genetic topics by using shape parameters smaller than one in the Gamma distribution priors. Sparsity is crucial to find meaningful, easy-tointerpret associations; those can be validated either through previous studies by looking in the literature, or subsequent tests in the lab.

Third, we present a nonparametric alternative model to [16] by replacing the continuous patient weights with a binary matrix whose probability distribution is induced by a hierarchical extension of the Indian buffet process (IBP). Also in the literature, the authors in [17] propose a nonparametric Poisson factorization model, but they rely on a stick-breaking construction different from the IBP, and the weights are continuous, which renders interpretability of the latent variables more tedious. The discrete nature of the IBP helps in terms of interpretability and allows combining the proposed Bayesian model with classical frequentist approaches for statistical testing between the inferred patient partitions. An efficient Markov chain Monte Carlo (MCMC) procedure based on a slice sampler for the hierarchical IBP is presented.

Bayesian modeling has already been proven useful for epistasis [53], pleiotropy [53, 52] or sub-phenotyping applications [35, 28]. To our knowledge, the proposed model is the first one to deal with clinical text data and genetic information jointly in a Bayesian nonparametric way, capturing phenotypic heterogeneity, epistasis and pleiotropy in a straightforward way while 
correcting for the cancer type as confounder. We consider multiple cancers jointly in order to increase statistical power, allow for the analysis of rare cancers, and identify fundamental mechanisms shared across different types of cancer.

\section{Methods}

\subsection{Study design and setting}

The study was designed as a retrospective cohort study for the development and analysis of techniques to analyze clinical narratives in the context of somatic mutations. It was performed at Memorial Sloan Kettering Cancer Center (MSKCC). The institutional review board of MSKCC provided a Waiver of Authorization (WOA; WA0426-13) for this study. Clinical notes were provided by the IT services group at MSKCC. The Center for Molecular Oncology at MSKCC provided the information about somatic mutations from the MSK Impact panels of patient tumors. We included 1,946 patients for which we had MSK Impact panel data and at least one clinical narrative available at the time of delivery. Data analyses were performed on the HPC compute systems at MSKCC. Additional statistical analyses were performed at University Carlos III and ETH Zürich.

\subsection{Database Description}

So far, genomic testing of tumors has been done routinely only for certain solid cancer tumors, such as melanoma, lung, or colon cancer. For most cancers, the available tests have been limited to analyzing one or a handful of genes at a time, and within each gene, only the most common mutations could be detected. ${ }^{1}$ A new targeted tumor sequencing test called MSK-IMPACT (Integrated Mutation Profiling of Actionable Cancer Targets) is able to detect somatic mutations and other critical somatic aberrations in both rare and common cancers $[10,51]$.

Using the MSK-IMPACT panels[51], somatic mutations regarding specific screened genes can be obtained as follows. For each patient, tumor cells are compared with healthy cells of that same patient, extracted from the blood stream, as illustrated in Figure 1. In this work, a gene is said to be mutated when there exists at least one difference in the sequence between the tumor cells and healthy cells for that particular gene. ${ }^{2}$ We finally obtain a binary matrix for $N=1946$ patients and $G=410$ genes where " 1 " encodes for a mutated gene and "0" otherwise. The screened genes have been shown to play a role in the development or behavior of tumors, although their individual relation to specific phenotypes remains obscure $[10,51]$.

Concerning the clinical information, based on all EHRs, we build a bag-of-word representation of unified medical language system (UMLS) terms, extracted using the Metamap ${ }^{3}$ processing tool [4]. The UMLS refers to a standardized, comprehensive thesaurus and ontology for biomedical concepts, whose objective is to provide facilities for natural signal processing tasks [7]. Since each patient can have a varying number of records, we group all clinical history into a single EHR, and only consider the appearance or absence of each UMLS term, i.e., binarized clinical features. We compute the tf-idf score for each UMLS term, and only keep the 300 clinical terms with highest score.

The database includes clinical and genetic information for $N=1946$ patients and 5 different cancer types: bladder cancer, breast carcinoma, colorectal cancer, non-small cell lung cancer, and prostate cancer. We consider genes and UMLS terms that are present in at least $1 \%$ of the patient population, resulting in $D=249$ dimensions, including 72 genes and 177 clinical terms.

\footnotetext{
${ }^{1}$ https: //www.mskcc.org/msk-impact

${ }^{2}$ The considered sequencing technology is able to remove most of the technical noise, in contrast to other technologies.

${ }^{3}$ Source code available at: https://metamap.nlm.nih.gov/
} 


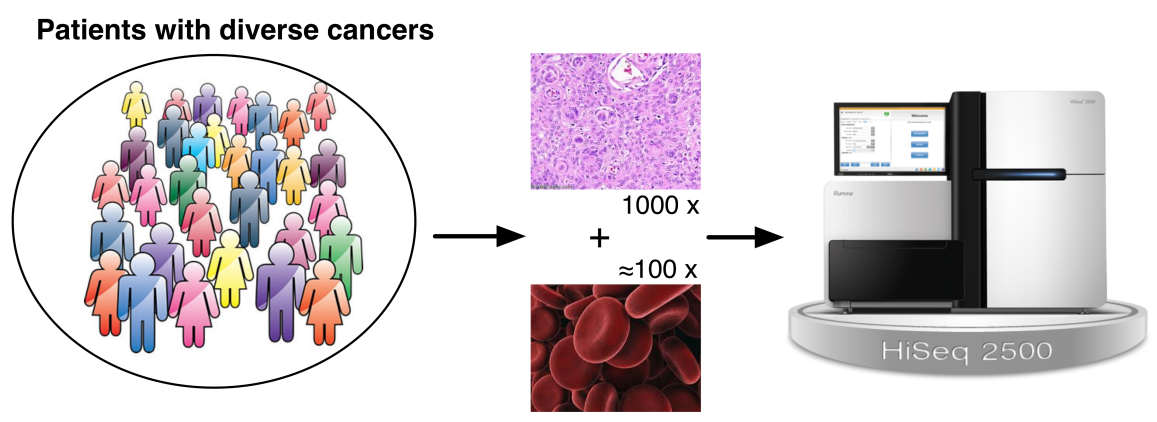

Figure 1: Diagram illustrating molecular profiling of tumors with the MSK-IMPACT Panel. (top) For each patient, tumor DNA is compared against normal tissue DNA in order to detect somatic mutations. The MSK-IMPACT panel is routinely used for a large number of patients per year at MSKCC [51].

Even if the dataset is binary, we can use a Poisson likelihood because of the high sparsity degree of such matrix (7.28\% of non-zero values). In such scenario, the Poisson distribution is a good approximation of a Bernoulli, and we adopt it by mathematical convenience in the inference process $[14,46]$. In the following, we use this data for: a) discovering latent factors and, b) testing for significant features (either genetic or phenotypical) associated to each factor.

\subsection{Classical Approach: Case-Control Setup}

The most common approach for genetic association studies is the case-control (CC) setup, which compares two large groups of individuals, one case group presenting a particular phenotype, and one control group without such phenotype.All individuals in each group are genotyped to identify somatic mutations in a panel of genes. For each of these genes, it is then investigated if the somatic mutation is significantly associated with the phenotype of interest. In such setups, the fundamental unit for reporting effect sizes is the odds ratio. The odds ratio in this case refers to the odds of exhibiting the phenotype for individuals having a specific somatic mutation and the odds of exhibiting the phenotype for individuals who do not present such somatic mutation. A $p$-value for the significance of the odds ratio is typically computed using a simple $\chi$-squared test or Fisher test. Finding odds ratios that are significantly different from one is the objective of an association study because this shows that there is statistical evidence that the somatic mutation is associated with the phenotype.

\subsection{Confounder Correcting Approach: Linear Mixed Model}

Linear mixed models (LMMs) have proved particularly useful for genetic association studies due to its capacity to account for confounding effects and limit the number of false associations [31, 30]. Let $\mathbf{X}$ be the observation matrix, where each element $x_{n g}$ corresponds to an indicator variable for a particular patient $n \in\{1, \ldots, N\}$ and somatic mutation in gene $g \in\{1, \ldots, G\}$, and $\boldsymbol{x}_{g} \in\{0,1\}^{N \times 1}$ is the indicator vector for gene $g$ across all patients. The binary variable $x_{n g}$ indicates whether any somatic mutation occurred in the corresponding gene. Let $y_{n q}$ be the binary indicator variable of the presence of a certain clinical feature $q \in\{1, \ldots, Q\}$ for a given patient $n$, and $\boldsymbol{y}_{q} \in \mathbb{R}^{N \times 1}$ the indicator vector for clinical feature $q$ across all patients. Finally, let us define $c_{n l}$ as the binary indicator variable of patient $n$ to the cancer type $\ell \in\{1, \ldots, L\}$, and $\boldsymbol{c}_{n} \in\{0,1\}^{1 \times L}$ the cancer type assignment vector, where $\sum_{\ell} c_{n \ell}=1$ (for simplicity, we only consider patients having one single type of cancer). For each pair of gene $g$ and clinical feature $q$, a LMM can be defined as follows: 


$$
\boldsymbol{y}_{q}=\boldsymbol{x}_{g} \beta_{q g}+\boldsymbol{u}_{q g}+\boldsymbol{\epsilon}_{q g}
$$

where $\beta_{q g} \in \mathbb{R}$ refer to the fixed effect of feature $q$ and gene $g$, and $\boldsymbol{u}_{q g}, \boldsymbol{\varepsilon}_{q g} \in \mathbb{R}^{N \times 1}$ are the random effects (structured noise and observational noise, respectively). The prior assumptions for the structured and uniform noises are $\boldsymbol{u}_{q g} \sim \mathcal{N}\left(0, \sigma_{u}^{2} \mathbf{K}\right)$ and $\varepsilon_{q g} \sim \mathcal{N}\left(0, \sigma_{e}^{2} \mathbf{I}\right)$, where $\mathbf{K}$ refers to a similarity matrix between the patients, for instance, the cosine similarity of the cancer type assignment vectors $\boldsymbol{c}_{i}$ and $\boldsymbol{c}_{j}, \mathbf{K}=\mathbf{C C}^{T}$. The LMM assumes that the output $\boldsymbol{y}_{\boldsymbol{q}}$ is Gaussian-distributed:

$$
\boldsymbol{y}_{q} \sim \mathcal{N}\left(\boldsymbol{x}_{g} \boldsymbol{\beta}_{q g}, \sigma_{u}^{2} \mathbf{K}+\sigma_{e}^{2} \mathbf{I}\right) .
$$

When the data is binary or count data, a common practice is to apply a standard rank-based inverse normal transformation beforehand as a preprocessing step [24], although this has become controversial more recently [5]. LMMs are discriminative since they try to model the conditional probability $p\left(\boldsymbol{y}_{q} \mid \boldsymbol{x}_{g}\right)$. In this paper, we propose an alternative generative approach that models the joint distribution $p\left(\boldsymbol{y}_{q}, \boldsymbol{x}_{g}\right)$ and captures complex correlations via latent factors.

\subsection{Hierarchical Bayesian Nonparametric Approach}

Let $\mathbf{X} \in \mathbb{N}^{N \times D}$ be the observation matrix of count data for $N$ patients and $D$ dimensions, where $D$ includes both clinical and genetic information, i.e., $D=G+Q$, where $G$ is the number of genes and $Q$ is the number of clinical terms. In the following, we propose two Poisson factor analysis (PFA) approaches to model the joint observation matrix $\mathbf{X}$ of genetic information and clinical data. In these models, patients will be represented by binary feature activation vectors, and each of these features will capture common correlation patterns among the somatic mutations and clinical term occurrences.

\subsubsection{Poisson Factor Analysis (PFA)}

We first consider a nonparametric non-negative matrix factorization model with Poisson likelihood and Gamma-distributed factors:

$$
\begin{aligned}
x_{n d} & \sim \operatorname{Poisson}\left(\mathbf{Z}_{n} \mathbf{A}_{d}\right), \\
\mathbf{A}_{k d} & \sim \operatorname{Gamma}\left(\alpha_{\mathbf{A}}, \frac{\mu_{\mathbf{A}}}{\alpha_{\mathbf{A}}}\right), \\
\mathbf{Z} & \sim \operatorname{IBP}(\alpha),
\end{aligned}
$$

where $\alpha$ is the concentration parameter of the IBP controlling the a priori number of ones in matrix $\mathbf{Z}$ (i.e., the a priori expected number of latent features), and $\mu_{\mathbf{A}}, \alpha_{\mathbf{A}}$ are the prior mean and shape parameter for each element of matrix $\mathbf{A}$. Sparsity can be induced easily in the factors by choosing $\alpha_{\mathbf{A}} \ll 1$. Inference is performed using an MCMC approach based on a semi-ordered stick-breaking representation of the IBP prior [44].

\subsubsection{Hierarchical Poisson Factor Analysis (H-PFA)}

Although different types of cancer are known to share similar phenotypes and underlying mechanisms (shared activation of certain pathways), the mutation rate and phenotype occurrence might vary in different proportions, according to each type of cancer. Given this premise, we propose a hierarchical Bernoulli process Poisson factor analysis model to allow for different feature activation levels depending on each type of cancer. In the following, we will shorten the name of this model to hierarchical Poisson factor analysis (H-PFA).

Let $r_{n} \in[1, \ldots, L]$ be a categorical variable indicating the type of cancer of patient $n$ among the total number of cancer types $L\left(r_{n}\right.$ corresponds to the index of the non-zero value in vector $\boldsymbol{c}_{n}$ defined in Section 2.4). A hierarchical construction can be formulated based on the finite representation of the IBP and letting $K \rightarrow \infty$, such that different levels of feature activation 
are allowed for each type of cancer. Let $\rho_{k}$ be the global activation probability of feature $k$, and $\pi_{k}^{\ell}$ be the specific activation probability of feature $k$ for cancer type $\ell \in[1, \ldots, L]$. We can then assume that each specific activation probability is Beta-distributed such that $\mathbb{E}_{\ell}\left[\pi_{k}^{\ell}\right]=\rho_{k}$ :

$$
\begin{array}{rlrl}
\rho_{k} & \sim \operatorname{Beta}\left(\frac{\alpha}{K}, 1\right) & z_{n k} & \sim \operatorname{Bernoulli}\left(\pi_{k}^{r_{n}}\right) \\
\pi_{k}^{\ell} \mid \rho_{k} & \sim \operatorname{Beta}\left(\frac{\rho_{k}}{1-\rho_{k}}, 1\right) & \mathbf{A}_{k d} & \sim \operatorname{Gamma}\left(\alpha_{\mathbf{A}}, \frac{\mu_{\mathbf{A}}}{\alpha_{\mathbf{A}}}\right) \\
\mathbf{x}_{n d} & \sim \operatorname{Poisson}\left(\mathbf{Z}_{n} \mathbf{A}_{d}\right),
\end{array}
$$

where the feature activation variables in vector $\mathbf{Z}_{n}$ are drawn from different activation probability vectors $\left\{\boldsymbol{\pi}^{1}, \ldots, \boldsymbol{\pi}^{L}\right\}$ depending on the type of cancer $r_{n}$ of patient $n$. When $K \rightarrow \infty$, this prior over $\mathbf{Z}$ is equivalent to a hierarchical Beta process (BP) construction [45] on top of Bernoulli processes (BePs) in the De Finetti representation. In the same way that a hierarchical Dirichlet process (HDP) allows for atom sharing with varying weights across different groups of data, the H-PFA allows for feature sharing with different activation weights across different types of cancer.

\subsection{Statistical Methodology}

Once the model has been trained (samples from an approximate posterior distribution can be drawn), we proceed with a classical frequentist approach ${ }^{4}$ to identify statistically significant clinico-genetic associations across cancer types. First, we take $M$ posterior samples from the posterior distribution of $\mathbf{Z}$ given $\mathbf{A}$ fixed. For each sample, patients that have the same feature assignment vector (activation pattern of features) can be grouped together in the same subpopulation. For instance, subpopulation (1001) refers to all patients having the first and fourth feature active. Let $P$ refer to the total number of inferred subpopulations across the $M$ posterior samples. By considering multiple posterior samples, we obtain slightly different partitions of patients in subpopulations. This can be seen as performing soft-clustering of patients, i.e., patients that are in-between subgroups might be assigned to different subpopulations in different posterior samples. Thus, the method is more robust against model inaccuracies at clustering patients. This is an important benefit of the Bayesian framework.

Next, to make our method robust against outliers (e.g., patients with rare features), we perform bootstrapping $B$ times for each subpopulation and posterior sample. Bootstrapping relies on random sampling with replacement. It is a technique used for computing robust estimators against outliers by sampling from an approximating distribution, which is particularly useful for hypothesis testing when the model assumptions are in doubt or unknown [50]. The standard bootstrapping approach relies on the construction of an estimator for hypothesis testing based on a number $B$ of resamples with replacement of the observed dataset (and of equal size to the observed dataset), i.e., sampling with replacement from the empirical distribution of the observed data.

Finally, given $M$ posterior samples and $B$ bootstrapping instances for each sample, we end up with $M B$ different subpopulation instances. Measures of effect size (quantitative measure of the difference between two subpopulations) and statistical significance can be computed for each instance and then averaged across them, so that partition inaccuracies and outlier effects are mitigated. To identify statistically significant dimensions for each latent feature $k=1, \ldots, K$ in sample $m$ and bootstrap $b$, we split the whole patient population in two subgroups, $\mathcal{S}_{k}(m, b)$ and $\mathcal{S}_{-k}(m, b)$, corresponding to patients whose latent feature $k$ is active or inactive respectively, and perform two-sample statistical tests for each dimension $d$.

\footnotetext{
${ }^{4}$ Note that statistical significance could also be accounted for using Bayesian factors or posterior predictive checks [13]. We here adopt the most established approach in the field for statistical significance.
} 


\subsubsection{Effect size}

For each latent feature $k$ and dimension $d$ (either clinical or genetic), we compute the effect size $\Delta_{k d}$ as:

$$
\Delta_{k d}=\mathbb{E}_{m, b}\left[\delta_{k d}(m, b)\right],
$$

where $\boldsymbol{\delta}_{k d}=\left\{\delta_{k d}(m, b)\right\}_{\forall m, b}$ is an $M \times B$ matrix of effect sizes for each posterior sample $m$ and bootstrap iteration $b$. The expectation is done across all posterior samples and bootstrapping iterations, which are equally probable. For each feature $k$ and input dimension $d$, we check for mean differences, i.e.,

$$
\delta_{k d}(m, b)=\mu_{d}\left(\mathcal{S}_{k}(m, b)\right)-\mu_{d}\left(\mathcal{S}_{-k}(m, b)\right),
$$

where $\mu_{d}(\mathcal{G})$ is the mean value of variable $d$ for a given subpopulation $\mathcal{G}$.

\subsubsection{Statistical significance}

To measure how significant an effect size $\delta_{k d}(m, b)$ is, for each posterior sample $m$ and bootstrap instance $b$, we compute a statistical significance value $v_{k d}(m, b)$ as the $p$-value resulting from a Fisher test, which is a standard test for discrete variables [50]. We define the $K \times D$ matrix of statistical significance $\boldsymbol{\Upsilon}$, for each latent feature $k$ and input dimension $d$ as the median $p$-value across the $M$ samples and $B$ bootstrapping instances:

$$
\Upsilon_{k d}=\operatorname{median}_{m, b}\left[v_{k d}(m, b)\right],
$$

where $\boldsymbol{v}_{k d}$ denote the $M \times B$ matrix of statistical significance values $v_{k d}(m, b)$ for each posterior sample $m$ and bootstrapping instance $b$. Finally, we follow the Benjamini Hochberg procedure for multiple hypothesis testing to adjust the statistical significance threshold $\alpha_{s}$ such that a certain false discovery rate (FDR) is guaranteed [6]. An input dimension $d$ (either clinical or genetic) is said to be statistically significant for latent feature $k$ if its significance value $\Upsilon_{k d}$ (the median $p$-value across posterior samples and bootstrapping instances) is smaller than the adjusted threshold, i.e., $\Upsilon_{k d}<\alpha_{s}$. The whole procedure is summarized in Algorithm 1.

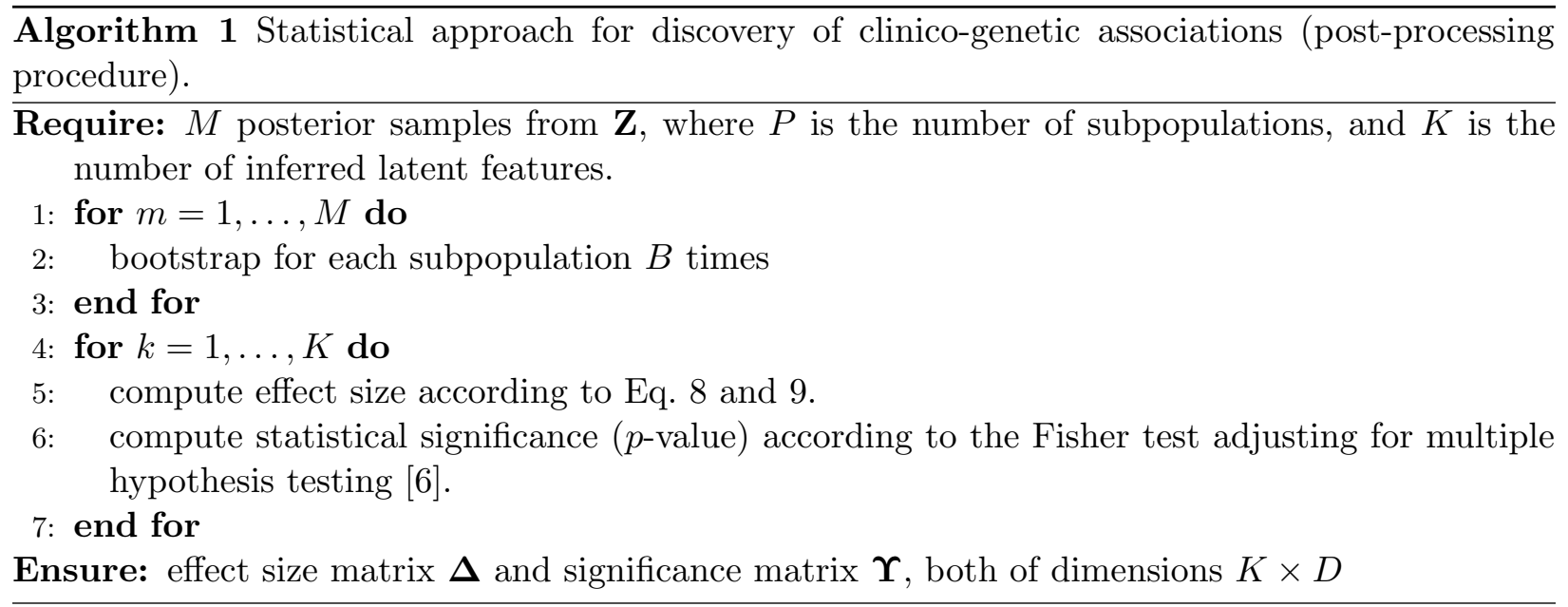

\subsection{Experimental Setup}

We compare the proposed H-PFA approach with a LMM and a standard case-control set-up for each potential clinico-genetic association. The model parameters for each LMM are found by 
maximizing the log likelihood using standard optimization techniques within a python platform called LIMIX [30]. In the final step, we obtain $p$-values for each pair $\left(\boldsymbol{y}_{q}, \boldsymbol{x}_{g}\right)$ using likelihood ratio tests. Regarding the case-control analysis, for each clinical term we consider a case and control group corresponding to the patients having that clinical term active or inactive respectively. Given such a partition, we perform an individual Fisher test for each gene. For all methods, we correct for multiple hypothesis testing based on the Benjamini-Hochberg approach [6]. To quantify the statistical significance of the features discovered by the H-PFA model, we follow the statistical procedure described in Section 2.6. To increase interpretability of the H-PFA model, we force one of the latent features to be active for all patients. This is a common practice in BNP models to capture mean effects [41, 47, 46], which in this case corresponds to phenotypical attributes common to all types of cancer. Finally, we have set the hyperparameters of the proposed H-PFA as $\alpha_{\mathbf{A}}=0.01$ and $\mu_{\mathbf{A}}=1$, while we infer the values for the concentration parameter $\alpha$.

\section{Results}

\subsection{Identification of Clinico-Genetic Associations}

Figure 2 represents the number of associations found by each method, and how many overlap across techniques. LMM found 14 clinico-genetic associations, CC found 178, and H-PFA found 95. ${ }^{5}$ LMM finds the least number of associations, since it corrects for the cancer type as a confounder effect, and only gets less well-known associations that are present across all types of cancer. CC discovered the highest number of associations, from which only $30 \%$ are shared with H-PFA. Out of the 95 associations discovered by the H-PFA approach, $63 \%$ were also present in any of the other methods. Figure 3 lists the associations that are shared across methods. Tables 1 and 2 present the list of clinico-genetic associations found by LMM and CC methods (for CC, we only report a random selection of associations, but the complete list can be found in the Appendix).

Next, Table 3 shows the list of inferred latent features by the H-PFA model. The bias term F0 reflects the high rate mutation of the TP53 gene which occur across all types of cancer. The TP53 gene is essential for the production of a protein called tumor protein p53. This protein acts as a tumor suppressor, which means that it regulates cell division by keeping cells from growing and dividing too fast or in an uncontrolled way. Because p53 is essential for regulating cell division and preventing tumor formation, it has been nicknamed the "guardian of the genome" [34]. On top of the bias term F0, H-PFA inferred 19 other latent features. Features F3, F5, and F17 capture complex phenotypes (no somatic mutations involved), whereas F4 and F18 mostly capture somatic mutations. Interestingly, F18 relates Esophagogastroduodenoscopy (a test to examine the lining of the esophagus, stomach, and the beginning of the small intestine) to multiple somatic mutations, which was already revealed by LMM in Table 2 . The remaining 14 features capture co-ocurrence of somatic mutations and clinical UMLS terms. Some latent features reflect well known relationships in oncology research. To name a few, mutations of gene PIK3CA (captured by F1 and F16) are present in over one-third of breast cancers; such mutations are nowadays known to be oncogenic and also implicated in cervical cancers [18]. Somatic mutations in the triad APC-KRAS-TP53 genes (captured by F0 and F6 together) are prominent in colon cancer [1]. Finally, previous studies have found direct physiological and molecular evidence for a role of gene FOXA1 in controlling cell proliferation in prostate cancer [23], which is accounted for in factor F12.

Figure 4 depicts the cancer-specific activation weights $\pi_{l}^{\ell}$ for each type of cancer $\ell$, as described in previous section. The activation of features present strong variations across cancer

\footnotetext{
${ }^{5}$ The H-PFA model is very flexible, as it can also find correlations between the genes, or between the clinical terms. 95 is the number of clinico-genetic associations only.
} 


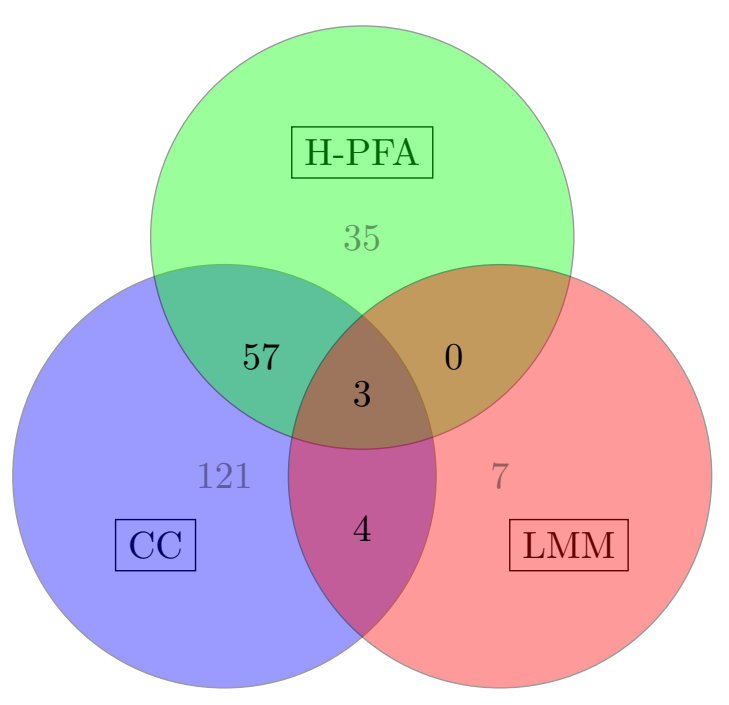

\begin{tabular}{|c|c|}
\hline Phenotype & Gene \\
\hline Stage IV Lung Adenocarcinoma & EGFR \\
\hline Adenocarcinoma of lung (disorder) & EGFR \\
\hline Malignant neoplasm of urinary bladder & TERT \\
\hline KRAS gene & KRAS \\
\hline Potassium Ion & KRAS \\
\hline Pulmonary function tests & STK11 \\
\hline $\begin{array}{l}\text { Rash and Dermatitis Adverse Event Associated } \\
\text { with Chemoradiation }\end{array}$ & TCF7L2 \\
\hline Colorectal Carcinoma & APC \\
\hline capecitabine & $\mathrm{APC}$ \\
\hline Colorectal & KRAS \\
\hline Lumpectomy of breast & PIK3CA \\
\hline Adriamycin & PIK3CA \\
\hline Renal function & ARID1A \\
\hline Simple mastectomy & GATA3 \\
\hline Malignant neoplasm of urinary bladder & TERT \\
\hline Hydronephrosis & KDM6A \\
\hline$\ldots$ & $\ldots$ \\
\hline
\end{tabular}

Figure 2: Venn Diagram of number of associations.

Figure 3: Shared associations across methods.

\begin{tabular}{llll}
\hline Phenotype & Gene & $\beta_{q g}$ & $p$-value \\
\hline \hline pump (device) & APC & 0.61 & $2.78 \mathrm{e}-53$ \\
S3 (sacral segmental innervation) & TP53 & 0.29 & $1.02 \mathrm{e}-09$ \\
Stage IV Lung Adenocarcinoma & EGFR & 0.32 & $2.07 \mathrm{e}-29$ \\
Folinic Acid-Fluorouracil-Irinotecan Regimen & APC & 0.59 & $1.08 \mathrm{e}-60$ \\
Folinic Acid-Fluorouracil-Irinotecan Regimen & KRAS & 0.30 & $1.16 \mathrm{e}-18$ \\
Hepatectomy & APC & 0.65 & $1.21 \mathrm{e}-52$ \\
Hepatectomy & KRAS & 0.27 & $7.27 \mathrm{e}-12$ \\
FOLFOX Regimen & APC & 0.66 & $5.12 \mathrm{e}-113$ \\
Tract & ARID1A & 0.21 & $5.61 \mathrm{e}-12$ \\
Malignant neoplasm of urinary bladder & TERT & 0.55 & $1.07 \mathrm{e}-61$ \\
Renal function & TERT & 0.28 & $8.40 \mathrm{e}-12$ \\
Flushing & APC & 0.30 & $1.54 \mathrm{e}-11$ \\
Non-Small Cell Lung Carcinoma & EGFR & 0.18 & $4.11 \mathrm{e}-11$ \\
Colorectal Carcinoma & APC & 0.61 & $2.34 \mathrm{e}-63$ \\
Adenocarcinoma of lung (disorder) & EGFR & 0.26 & $1.49 \mathrm{e}-22$ \\
Simple mastectomy & PIK3CA & 0.16 & $3.40 \mathrm{e}-08$ \\
Immunotherapy & TERT & 0.23 & $2.76 \mathrm{e}-12$ \\
Imodium & APC & 0.30 & $6.51 \mathrm{e}-15$ \\
capecitabine & APC & 0.30 & $1.94 \mathrm{e}-15$ \\
Pulmonary function tests & STK11 & 0.16 & $2.19 \mathrm{e}-09$ \\
\hline
\end{tabular}

Table 1: Subset of clinico-genetic associations found using the CC setup. A complete list can be found in the Appendix.

types. Some features are clearly cancer-specific (F1 and F3 typically activate for breast carcinoma patients; F6, F11 and F15 are typically active for colorectal cancer; F7, F8 and F10 are almost exclusively active for non-small cell lung cancer, etc.), whereas other factors occur in similar proportions across cancers, e.g., feature F5 which capture typical adverse effects that manifest for all types of cancer (Prednisone is a synthetic corticosteroid drug which is regularly used to treat certain types of cancer, but has significant adverse effects).

Tables 4, 5, 7,8, and 9 show statistically-significant group associations across different somatic mutations and UMLS terms. For each clinical and genetic term, we give both the effect size 
bioRxiv preprint doi: https://doi.org/10.1101/623215; this version posted April 30, 2019. The copyright holder for this preprint (which was not certified by peer review) is the author/funder, who has granted bioRxiv a license to display the preprint in perpetuity. It is made available under aCC-BY 4.0 International license.

\begin{tabular}{llll}
\hline Phenotype & Gene & $\beta_{q g}$ & $p$-value \\
\hline \hline Stage IV Lung Adenocarcinoma & EGFR & 0.14 & $9.90 \mathrm{e}-14$ \\
Pulmonary function tests & STK11 & 0.11 & $5.67 \mathrm{e}-06$ \\
Esophagogastroduodenoscopy & ERBB3 & 0.10 & $4.62 \mathrm{e}-06$ \\
Adenocarcinoma of lung (disorder) & EGFR & 0.09 & $3.20 \mathrm{e}-06$ \\
Rash and Dermatitis Adverse Event Associated with Chemoradiation & TCF7L2 & 0.09 & $1.57 \mathrm{e}-04$ \\
Atrophic & PTEN & 0.09 & $2.06 \mathrm{e}-05$ \\
Esophagogastroduodenoscopy & ALK & 0.09 & $1.43 \mathrm{e}-05$ \\
Stage level 2 & ERBB4 & 0.08 & $3.34 \mathrm{e}-05$ \\
Positive Surgical Margin & EP300 & 0.08 & $1.43 \mathrm{e}-04$ \\
Esophagogastroduodenoscopy & CDH1 & 0.08 & $1.33 \mathrm{e}-04$ \\
Esophagogastroduodenoscopy & FLT4 & 0.08 & $4.15 \mathrm{e}-05$ \\
Malignant neoplasm of urinary bladder & TERT & 0.08 & $7.91 \mathrm{e}-05$ \\
Potassium Ion & KRAS & 0.08 & $6.72 \mathrm{e}-06$ \\
KRAS gene & KRAS & 0.07 & $9.76 \mathrm{e}-05$ \\
\hline
\end{tabular}

Table 2: Clinico-genetic associations found using the LMM approach. The associations have been sorted according to the effect size $\beta_{q g}$ which refers to the linear weight of the regression, as described in Section 2.4.
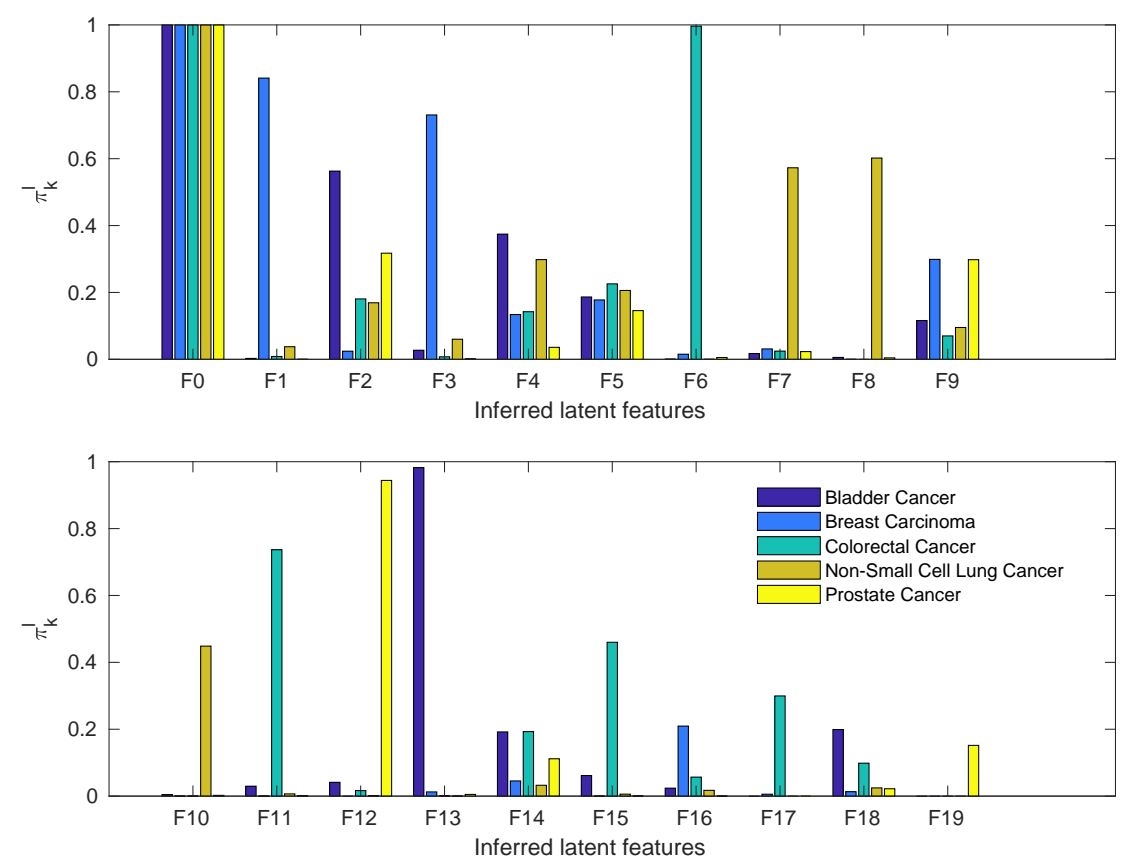

Figure 4: Activation weights $\pi_{k}^{\ell}$ for each cancer type $\ell$ inferred by H-PFA.

and significance. Our method is able to provide concise grouping of both clinical terms and somatic mutations. Among the clinical terms, we find both phenotypical terms, as well as names of chemotherapy medications (Adriamycin, Irinotecan, or Leucovorin). Table 4 shows cancer-specific clinico-genetic associations. We recover well-known associations (such as APC gene mutation being prominant in colorectal cancer, or STK11 to lung carcinoma), but other associations are more surprising, such as GATA3 gene with bone mineral density.

Finally, H-PFA found several statistically significant sets of associations involving somatic mutations in gene TERT, as shown in Table 5. Somatic mutations in the gene promoter of telomerase reverse transcriptase (TERT) have been found in $70-79 \%$ of bladder tumors in a multi-institutional study published in European Urology [36]. Table 5 shows that TERT muta- 


\begin{tabular}{|c|c|c|c|}
\hline Feat. & $m_{k}$ & Phenotypes & Genes \\
\hline F1. & 460 & Simple mastectomy $(0.17)$, Xeloda $(0.15)$, Lumpectomy of breast $(0.12)$, capecitabine $(0.09)$ & PIK3CA (0.31) \\
\hline F2. & 402 & $\begin{array}{l}\text { Renal function (0.29), Coronary Artery Disease (0.28), Stent, device (0.22), cardiologist } \\
(0.21) \text {, Urology }(0.20) \text {, Hydronephrosis }(0.16)\end{array}$ & MTOR (0.04) \\
\hline F3. & 400 & $\begin{array}{l}\text { Invasive Ductal Breast Carcinoma (0.57), axillary lymph node dissection (0.38), Simple mas- } \\
\text { tectomy (0.37), Noninfiltrating Intraductal Carcinoma (0.36), Lumpectomy of breast }(0.33) \text {, } \\
\text { Adriamycin }(0.29)\end{array}$ & None \\
\hline F4. & 392 & None & $\begin{array}{l}\text { ETV6 }(0.22), \text { PT- } \\
\text { PRD }(0.19), \text { ATR } \\
(0.19), \quad \text { PTPRT } \\
(0.17),, \ldots\end{array}$ \\
\hline$\overline{\text { F5. }}$ & 361 & $\begin{array}{l}\text { Entire intercostal space }(0.22) \text {, Midclavicular line }(0.21) \text {, Per Minute }(0.20) \text {, Prednisone } \\
(0.19) \text {, Upper Extremity }(0.19) \text {, Entire head }(0.18) \text {, Dizziness }(0.17) \text {, Redness }(0.17) \text {, Serum } \\
(0.17) \text {, Bedtime (qualifier value) }(0.16), \ldots\end{array}$ & None \\
\hline F7. & 350 & $\begin{array}{l}\text { Lobectomy (0.48), Pulmonary function tests (0.29), Thoracotomy }(0.27) \text {, Non-Small Cell } \\
\text { Lung Carcinoma }(0.27)\end{array}$ & EGFR (0.09) \\
\hline F8. & 326 & $\begin{array}{l}\text { Non-Small Cell Lung Carcinoma (0.13), Stage IV Lung Adenocarcinoma (0.10), natural } \\
\text { daughter - RoleCode }(0.09) \text {, pemetrexed }(0.08)\end{array}$ & $\begin{array}{lr}\text { KRAS } & (0.36) \\
\text { STK11 } & (0.26), \\
\text { KEAP1 } & (0.20)\end{array}$ \\
\hline F9. & 326 & $\begin{array}{l}\text { Lytic lesion (0.29), Zometa }(0.27) \text {, Fracture }(0.25) \text {, Sclerosis }(0.25) \text {, Bone Lesion }(0.23) \text {, Bone } \\
\text { structure of sacrum }(0.23) \text {, Hip arthralgia }(0.23) \text {, Bone structure of ilium }(0.19) \text {, Palliative } \\
\text { Care }(0.17)\end{array}$ & $\operatorname{ATRX}(0.04)$ \\
\hline F10. & 266 & $\begin{array}{l}\text { Stage IV Lung Adenocarcinoma (0.62), pemetrexed (0.61), Adenocarcinoma of lung (disor- } \\
\text { der) }(0.60) \text {, mediastinal lymphadenopathy }(0.35)\end{array}$ & $\begin{array}{l}\text { EGFR (0.30), TP53 } \\
(0.18)\end{array}$ \\
\hline F11. & 265 & $\begin{array}{l}\text { FOLFOX Regimen (0.54), KRAS gene (0.45), Folinic Acid-Fluorouracil-Irinotecan Regimen } \\
(0.43) \text {, Leucovorin }(0.43) \text {, irinotecan }(0.39) \text {, Colorectal Carcinoma (0.39), Cold intolerance } \\
(0.37) \text {, Midclavicular line }(0.27) \text {, Sigmoid colon }(0.27) \text {, Colorectal }(0.27)\end{array}$ & $\begin{array}{l}\text { PTPRT }(0.05) \\
\text { CARD11 }(0.04)\end{array}$ \\
\hline F15. & 159 & $\begin{array}{l}\text { Rectum (0.45), Rash and Dermatitis Adverse Event Associated with Chemoradiation (0.28), } \\
\text { capecitabine }(0.26) \text {, Node stage N0 }(0.23)\end{array}$ & $\begin{array}{ll}\text { APC } & (0.14) \\
\text { TCF7L2 } & (0.14), \\
\text { TSC2 }(0.09) & \end{array}$ \\
\hline F16. & 149 & $\begin{array}{l}\text { Consistency (0.55), Vagina (0.50), Clinic / Center - Mobile (0.43), Bilateral Salpingectomy } \\
\text { with Oophorectomy (0.39), Atrophic (0.39), New medications (0.35), Personal Attribute } \\
(0.32) \text {, Uterus (0.31), Ovarian (0.30), Bone Mineral Density Test (0.30), Ovary }(0.29)\end{array}$ & $\begin{array}{ll}\text { PIK3CA } & (0.12), \\
\text { PTEN }(0.07)\end{array}$ \\
\hline F17. & 107 & $\begin{array}{l}\text { Depression motion (0.95), Structure of long bone (0.82), S3 (sacral segmental innervation) } \\
\text { (0.82), pump (device) (0.79), intrahepatic (0.78), Pulse taking (0.74), Midclavicular line } \\
(0.73) \text {, Entire intercostal space (0.70), Hepatectomy (0.62), Flowcharts (Computer) }(0.57)\end{array}$ & None \\
\hline F18. & 95 & Esophagogastroduodenoscopy $(0.12)$ & $\begin{array}{l}\text { POLE }(0.65), \text { ROS1 } \\
(0.61), \quad \text { DNMT1 } 1 \\
(0.59), \text { ATR }(0.58), \\
\text { ATM }(0.57), \\
(0.54), \ldots\end{array}$ \\
\hline F19. & 41 & Optic Nerve (0.90), Gross hematuria (0.90), Dyspepsia (0.57), Lupron (0.45) & $\mathrm{AR}(0.22)$ \\
\hline
\end{tabular}

Table 3: Latent features inferred by H-PFA. We depict the UMLS terms and genes with highest weights separately, up until the weight decays more than $50 \% . m_{k}$ is the number of patients with each feature active. 
$100 \%$ patients with breast carcinoma

\begin{tabular}{|c|c|c|c|c|c|}
\hline \multicolumn{3}{|l|}{ Clinical record } & \multicolumn{3}{|c|}{ Genetic information } \\
\hline Phenotype $q$ & $A_{k q}$ & $p$-value & Gene $g$ & $A_{k g}$ & $p$-value \\
\hline Invasive Ductal Breast Carcinoma & 0.64 & $3.54 \mathrm{e}-49$ & PIK3CA & 0.22 & $4.38 \mathrm{e}-07$ \\
\hline Simple mastectomy & 0.39 & $4.26 \mathrm{e}-22$ & GATA3 & 0.12 & $3.08 \mathrm{e}-05$ \\
\hline Noninfiltrating Intraductal Carcinoma & 0.34 & $2.02 \mathrm{e}-20$ & & & \\
\hline Lumpectomy of breast & 0.32 & $3.94 \mathrm{e}-17$ & & & \\
\hline axillary lymph node dissection & 0.28 & $3.43 \mathrm{e}-16$ & & & \\
\hline Fishes & 0.22 & $1.01 \mathrm{e}-10$ & & & \\
\hline Adriamycin & 0.21 & $4.09 \mathrm{e}-11$ & & & \\
\hline Bone Mineral Density Test & 0.15 & $2.19 \mathrm{e}-06$ & & & \\
\hline
\end{tabular}

$100 \%$ patients with non-small cell lung cancer

\begin{tabular}{|c|c|c|c|c|c|}
\hline \multicolumn{3}{|l|}{ Clinical record } & \multicolumn{3}{|c|}{ Genetic information } \\
\hline Phenotype $q$ & $A_{k q}$ & $p$-value & Gene $g$ & $A_{k g}$ & $p$-value \\
\hline FOLFOX Regimen & 0.80 & $1.52 \mathrm{e}-29$ & $\mathrm{APC}$ & 0.63 & $3.50 \mathrm{e}-17$ \\
\hline Colorectal & 0.37 & $4.04 \mathrm{e}-09$ & TP53 & 0.42 & $3.50 \mathrm{e}-08$ \\
\hline Sigmoid colon & 0.35 & $1.88 \mathrm{e}-09$ & & & \\
\hline Colorectal Carcinoma & 0.33 & $8.49 \mathrm{e}-08$ & & & \\
\hline Cold intolerance & 0.32 & $2.03 \mathrm{e}-08$ & & & \\
\hline irinotecan & 0.29 & $8.72 \mathrm{e}-08$ & & & \\
\hline Leucovorin & 0.29 & $4.90 \mathrm{e}-07$ & & & \\
\hline $\begin{array}{l}\text { Folinic Acid-Fluorouracil-Irinotecan } \\
\text { Regimen }\end{array}$ & 0.25 & $1.19 \mathrm{e}-05$ & & & \\
\hline Hepatectomy & 0.23 & $3.35 \mathrm{e}-06$ & & & \\
\hline
\end{tabular}

$97 \%$ patients with breast carcinoma, $3 \%$ with non-small cell lung cancer

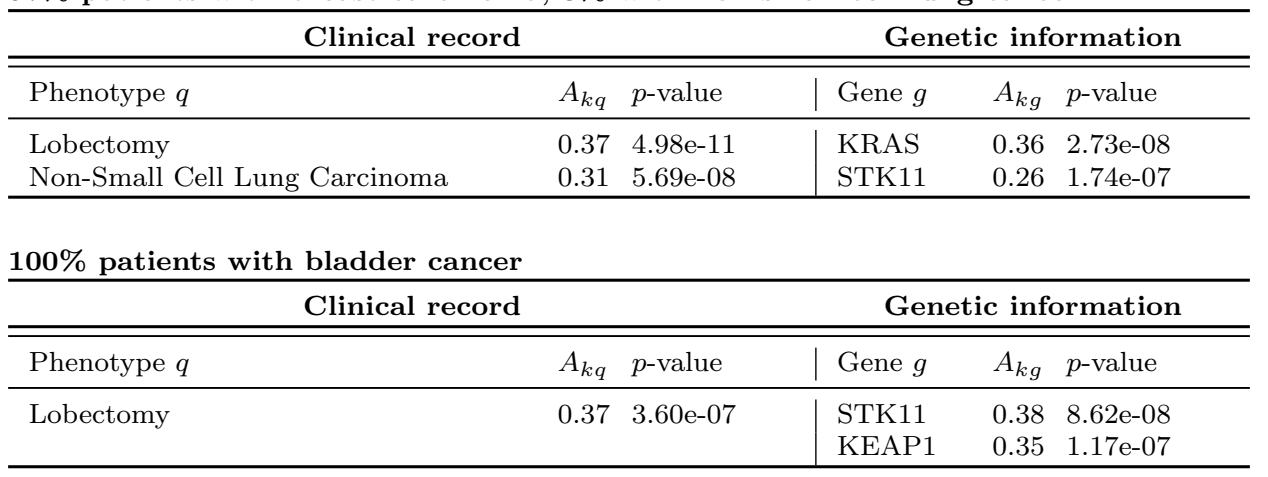

Table 4: Clinico-genetic associations found by the H-PFA (1/2). These tables depict the statistically significant clinical and genetic features associated to the latent factors listed in Table 3, after applying the statistical methodology described in Section 2.6. On top of each table, we describe the distribution of cancer types of patients for which the association is active. 
$100 \%$ patients with colorectal cancer

\begin{tabular}{lll|lll}
\hline \multicolumn{2}{c}{ Clinical record } & \multicolumn{3}{c}{ Genetic information } \\
\hline \hline Phenotype $q$ & $A_{k q}$ & $p$-value & Gene $g$ & $A_{k g}$ & $p$-value \\
\hline Tract & $\mathbf{0 . 3 7}$ & $\mathbf{3 . 3 9 e - 0 8}$ & TERT & $\mathbf{0 . 5 7}$ & $\mathbf{4 . 5 6 e - 1 2}$ \\
$\begin{array}{l}\text { Malignant neoplasm of urinary } \\
\text { bladder }\end{array}$ & $\mathbf{0 . 3 6}$ & $\mathbf{3 . 9 2 e - 0 7}$ & FGFR3 & 0.50 & $4.15 \mathrm{e}-13$ \\
Gross hematuria & $\mathbf{0 . 3 3}$ & $\mathbf{2 . 6 7 e - 0 6}$ & & & \\
\hline
\end{tabular}

$100 \%$ patients with prostate cancer

\begin{tabular}{lll|llll}
\hline \multicolumn{3}{c}{ Clinical record } & \multicolumn{3}{c}{ Genetic information } \\
\hline \hline Phenotype $q$ & $A_{k q}$ & $p$-value & Gene $g$ & $A_{k g}$ & $p$-value \\
\hline Gross hematuria & $\mathbf{0 . 7 4}$ & $\mathbf{1 . 8 1 e - 2 0}$ & TERT & $\mathbf{0 . 5 8}$ & $\mathbf{5 . 5 9 e - 1 2}$ \\
$\begin{array}{l}\text { Malignant neoplasm of urinary } \\
\text { bladder }\end{array}$ & $\mathbf{0 . 4 1}$ & $\mathbf{2 . 8 0 e - 0 8}$ & KDM6A & 0.31 & $6.48 \mathrm{e}-06$ \\
Tract & & & & & & \\
chain of objects & $\mathbf{0 . 3 9}$ & $\mathbf{2 . 0 8 e - 0 8}$ & & & \\
Hydronephrosis & 0.32 & $1.92 \mathrm{e}-06$ & & & & \\
\hline
\end{tabular}

$100 \%$ patients with non-small cell lung cancer

\begin{tabular}{|c|c|c|c|c|}
\hline \multicolumn{2}{|l|}{ Clinical record } & \multicolumn{3}{|c|}{ Genetic information } \\
\hline Phenotype $q$ & $A_{k q} \quad p$-value & Gene $g$ & $A_{k g}$ & $p$-value \\
\hline Gross hematuria & $0.39 \quad 1.72 \mathrm{e}-07$ & TERT & 0.64 & $4.86 e-13$ \\
\hline $\begin{array}{l}\text { Malignant neoplasm of urinary } \\
\text { bladder }\end{array}$ & $0.379 .85 \mathrm{e}-07$ & FGFR3 & 0.50 & $1.97 \mathrm{e}-11$ \\
\hline Tract & $0.302 .19 \mathrm{e}-05$ & $\begin{array}{l}\text { KDM6A } \\
\text { CREBBP }\end{array}$ & $\begin{array}{l}0.36 \\
0.32\end{array}$ & $\begin{array}{l}1.20 \mathrm{e}-06 \\
3.19 \mathrm{e}-06\end{array}$ \\
\hline
\end{tabular}

$100 \%$ patients with bladder cancer

\begin{tabular}{lll|lll}
\hline \multicolumn{2}{c}{ Clinical record } & \multicolumn{3}{c}{ Genetic information } \\
\hline \hline Phenotype $q$ & $A_{k q}$ & $p$-value & Gene $g$ & $A_{k g}$ & $p$-value \\
\hline $\begin{array}{l}\text { Malignant neoplasm of urinary } \\
\text { bladder }\end{array}$ & $\mathbf{0 . 5 6}$ & $\mathbf{4 . 2 4 e - 1 2}$ & TERT & $\mathbf{0 . 6 7}$ & $\mathbf{1 . 7 9 e - 1 4}$ \\
Tract & & & & & \\
Renal function & $\mathbf{0 . 4 6}$ & $\mathbf{2 . 0 0 e - 1 0}$ & ARID1A & 0.45 & $2.32 \mathrm{e}-08$ \\
Gross hematuria & 0.45 & $5.51 \mathrm{e}-11$ & & & \\
\hline
\end{tabular}

Table 5: Clinico-genetic associations found by the H-PFA (2/2). All these associations involve gene TERT. One same set (depicted in bold) appears in all associations.

tions are associated to not only malignant neoplasm of urinary bladder (which is not surprising), but also hematuria and hydronephrosis. Hematuria refers to the presence of red blood cells in the urine. Also, hydronephrosis is a condition that typically occurs when the kidney swells due to the failure of normal drainage of urine from the kidney to the bladder. Hydronephrosis is not a primary disease, but results from some other underlying disease (cancer in this case) as the result of a blockage or obstruction in the urinary tract. H-PFA points out to interesting gene relationships (KDM6A, CREBBP, and ARID1A genes) with TERT, which have been partially studied in the literature [33, 39, 25]. 


\section{Conclusion}

This paper proposes a novel Bayesian nonparametric approach for discovering clinico-genetic associations between somatic mutations and EHR-based clinical features. We present a hierarchical Bernoulli process Poisson factor analysis model based on a hierarchical construction of Beta processes and Bernoulli processes. Our approach is not specific to cancer data nor Electronic Health Records, but can be broadly used to discover associations between arbitrary count data features. Compared to other approaches, our model delivers group-associations instead of pairwise ones, accounting for epistatic and pleiotropical effects straightforwardly. The delivered associations are statistically significant after correction for multiple hypothesis testing combined with a bootstrapping procedure, to better account for false positives. These associations give potentially interesting insights for future research in oncology. Under the proposed model, we hopefully open the door to find new associations that give rise to hypotheses, and if those are validated, then we may get new insights about cancer biology. Ultimately, studies like this one have the potential to lead us towards more accurate diagnosis, and inform us about actionable pathways when considering cancer therapy, where interventions through drug administration can be designed.

\section{Acknowledgements}

This study was supported by the MSK Cancer Center Support Grant (P30 CA008748). M.F.P. was supported by the European Union 7th Framework Programme through the Marie Curie Initial Training Network "Machine Learning for Personalized Medicine" MLPM2012, Grant No. 316861 (to F.P.C and G.R.). This work was also partially supported by MINECO/FEDER ('ADVENTURE', id. TEC2015-69868-C2-1-R), and Comunidad de Madrid (project 'CASICAM-CM', id. S2013/ICE-2845). We gratefully acknowledge helpful discussions with Theofanis Karaletsos, Chris Sander, and Niki Schultz. Moreover, we would like to thank Iker Huerga, Chris Crosbie, Stuart Gardos and David Artz for supporting the work with data deliveries from the clinical data warehouse.

\section{References}

[1] Lauri A. Aaltonen, Paivi Peltomaki, Fredrick S. Leach, Pertti Sistonen, Lea Pylkkanen, Jukka-Pekka Mecklin, Heikki Jarvinen, Steven M. Powell, Jin Jen, Stanley R. Hamilton, et al. Clues to the pathogenesis of familial colorectal cancer. Science, 260(5109):812-817, May 1993.

[2] Tomasz Adamusiak and Mary Shimoyama. EHR-based phenome wide association study in pancreatic cancer. AMIA Summits on Translational Science Proceedings, 2014:9-15, April 2014.

[3] Ulrika Andersson, Roberta McKean-Cowdin, Ulf Hjalmars, and Beatrice Malmer. Genetic variants in association studies-review of strengths and weaknesses in study design and current knowledge of impact on cancer risk. Acta Oncologica (Stockholm, Sweden), 48(7):948-954, 2009.

[4] Alan R. Aronson. Effective mapping of biomedical text to the UMLS metathesaurus: the MetaMap program. In Proceedings of the AMIA Symposium, p. 17. American Medical Informatics Association, 2001.

[5] T Mark Beasley, Stephen Erickson, and David B Allison. Rank-based inverse normal transformations are increasingly used, but are they merited? Behavior genetics, 39(5):580, 2009 . 
[6] Yoav Benjamini and Yosef Hochberg. Controlling the false discovery rate: A practical and powerful approach to multiple testing. Journal of the Royal Statistical Society. Series B (Methodological), 57(1):289-300, January 1995.

[7] Olivier Bodenreider. The Unified Medical Language System (UMLS): Integrating biomedical terminology. Nucleic Acids Research, 32(suppl_1):D267-D270, January 2004.

[8] Claudia Calabrese, Natalie R Davidson, Nuno A Fonseca, Yao He, André Kahles, KjongVan Lehmann, Fenglin Liu, Yuichi Shiraishi, Cameron M Soulette, Lara Urban, et al. Genomic basis for rna alterations revealed by whole-genome analyses of 27 cancer types. bioRxiv, 2018.

[9] Katherine Redfield Chan, Xinghua Lou, Theofanis Karaletsos, Christopher Crosbie, Stuart M. Gardos, David Artz, and Gunnar Rätsch. An empirical analysis of topic modeling for mining cancer clinical notes. In 13th IEEE International Conference on Data Mining Workshops, ICDM Workshops, TX, USA, December 7-10, 2013, pp. 56-63, 2013.

[10] Donavan T. Cheng, Talia N. Mitchell, Ahmet Zehir, Ronak H. Shah, Ryma Benayed, Aijazuddin Syed, Raghu Chandramohan, Zhen Yu Liu, Helen H. Won, et al. Memorial Sloan Kettering-Integrated Mutation Profiling of Actionable Cancer Targets (MSK-IMPACT): A hybridization capture-based next-generation sequencing clinical assay for solid tumor molecular oncology. The Journal of molecular diagnostics: JMD, 17(3):251-264, May 2015.

[11] Aubrey D. N. J. de Grey. Protagonistic pleiotropy: Why cancer may be the only pathogenic effect of accumulating nuclear mutations and epimutations in aging. Mechanisms of Ageing and Development, 128(7-8):456-459, August 2007.

[12] Douglas F. Easton and Rosalind A. Eeles. Genome-wide association studies in cancer. Human Molecular Genetics, 17(R2):R109-R115, October 2008.

[13] Andrew Gelman, John B. Carlin, Hal S. Stern, David B. Dunson, Aki Vehtari, and Donald B. Rubin. Bayesian Data Analysis, volume 2. CRC press Boca Raton, FL, 2014.

[14] Prem Gopalan, Laurent Charlin, and David M. Blei. Content-based recommendations with Poisson factorization. In Advances in Neural Information Processing Systems 27, pp. 3176-3184. 2014.

[15] Prem Gopalan, Jake M. Hofman, and David M. Blei. Scalable recommendation with Poisson factorization. 2013.

[16] Prem Gopalan, Jake M. Hofman, and David M. Blei. Scalable recommendation with hierarchical Poisson factorization. In Proceedings of the Thirti-first Conference Annual Conference on Uncertainty in Artificial Intelligence (UAI-15). AUAI Press, 2015.

[17] Prem Gopalan, Francisco J. R. Ruiz, Rajesh Ranganath, and David M. Blei. Bayesian nonparametric Poisson factorization for recommendation systems. Artificial Intelligence and Statistics (AISTATS), 33:275-283, 2014.

[18] Arnaud Guille, Max Chaffanet, and Daniel Birnbaum. Signaling pathway switch in breast cancer. Cancer Cell International, 13(1):66, 2013.

[19] Douglas Hanahan and Robert A. Weinberg. The hallmarks of cancer. Cell, 100(1):57-70, January 2000.

[20] Douglas Hanahan and Robert A. Weinberg. Hallmarks of cancer: the next generation. Cell, 144(5):646-674, March 2011.

[21] Ricardo Henao, James T. Lu, Joseph E. Lucas, Jeffrey Ferranti, and Lawrence Carin. Electronic health record analysis via deep Poisson factor models. Journal of Machine Learning Research, 2015.

[22] N. Lynn Henry and Daniel F. Hayes. Cancer biomarkers. Molecular Oncology, 6(2):140-146, April 2012. 
[23] Yusuke Imamura, Shinichi Sakamoto, Takumi Endo, Takanobu Utsumi, Miki Fuse, Takahito Suyama, Koji Kawamura, Takashi Imamoto, Kojiro Yano, Katsuhiro Uzawa, et al. FOXA1 promotes tumor progression in prostate cancer via the insulin-like growth factor binding protein 3 pathway. PLoS ONE, 7(8):e42456, 2012.

[24] Ronald L. Iman and William J. Conover. The use of the rank transform in regression. Technometrics, 21(4):499-509, 1979.

[25] Sumit Isharwal, François Audenet, Eugene J. Pietzak, Eugene K. Cha, Gopa Iyer, Ahmet Zehir, Barry S. Taylor, Michael F. Berger, Satish Tickoo, Victor E. Reuter, et al. Comparison of genomic alterations in bladder urothelial tumors with and without telomerase reverse transcriptase promoter mutation using a next-generation sequencing assay, 2017.

[26] Peter B. Jensen, Lars J. Jensen, and Søren Brunak. Mining electronic health records: Towards better research applications and clinical care. Nature Reviews Genetics, 13(6):395405, 2012.

[27] André Kahles, Kjong-Van Lehmann, Nora C Toussaint, Matthias Hüser, Stefan G Stark, Timo Sachsenberg, Oliver Stegle, Oliver Kohlbacher, Chris Sander, Samantha J CaesarJohnson, et al. Comprehensive analysis of alternative splicing across tumors from 8,705 patients. Cancer cell, 34(2):211-224, 2018.

[28] David Knowles and Zoubin Ghahramani. Nonparametric Bayesian sparse factor models with application to gene expression modeling. The Annals of Applied Statistics, 5(2B):15341552, June 2011.

[29] Emilie Lalonde, Adrian S. Ishkanian, Jenna Sykes, Michael Fraser, Helen Ross-Adams, Nicholas Erho, Mark J. Dunning, Silvia Halim, Alastair D Lamb, et al. Tumour genomic and microenvironmental heterogeneity for integrated prediction of 5-year biochemical recurrence of prostate cancer: A retrospective cohort study. The Lancet Oncology, 15(13):1521-1532, December 2014.

[30] Christoph Lippert, Francesco Paolo Casale, Barbara Rakitsch, and Oliver Stegle. LIMIX: Genetic analysis of multiple traits. bioRxiv, p. 003905, May 2014.

[31] Christoph Lippert, Jennifer Listgarten, Ying Liu, Carl M. Kadie, Robert I. Davidson, and David Heckerman. FaST linear mixed models for genome-wide association studies. Nature Methods, 8(10):833-835, October 2011.

[32] Stéphane M. Meystre, Guergana K. Savova, Karin C. Kipper-Schuler, John F. Hurdle, and others. Extracting information from textual documents in the electronic health record: A review of recent research. Yearb Med Inform, 35:128-44, 2008.

[33] Michael L. Nickerson, Garrett M. Dancik, Kate M. Im, Michael G. Edwards, Sevilay Turan, Joseph Brown, Christina Ruiz-Rodriguez, Charles Owens, James C. Costello, Guangwu Guo, et al. Concurrent alterations in TERT, KDM6A, and the BRCA pathway in bladder cancer. Clinical Cancer Research, 20(18):4935-4948, 2014.

[34] Magali Olivier, Monica Hollstein, and Pierre Hainaut. TP53 mutations in human cancers: Origins, consequences, and clinical use. Cold Spring Harbor perspectives in biology, 2(1):a001008, 2010.

[35] Leopold Parts, Oliver Stegle, John Winn, and Richard Durbin. Joint genetic analysis of gene expression data with inferred cellular phenotypes. PLoS Genetics, 7(1):e1001276, January 2011.

[36] Sarah Payton. Bladder cancer: Mutation found in $>70 \%$ of tumours. Nature Reviews Urology, 10(11):616-616, 2013.

[37] Paul D. P. Pharoah, Alison M. Dunning, Bruce A. J. Ponder, and Douglas F. Easton. Association studies for finding cancer-susceptibility genetic variants. Nature Reviews Cancer, 4(11):850-860, November 2004. 
[38] Elsa Quintana, Mark Shackleton, Hannah R. Foster, Douglas R. Fullen, Michael S. Sabel, Timothy M. Johnson, and Sean J. Morrison. Phenotypic heterogeneity among tumorigenic melanoma cells from patients that is reversible and not hierarchically organized. Cancer Cell, 18(5):510-523, November 2010.

[39] Yohan Suryo Rahmanto, Jin-Gyoung Jung, Ren-Chin Wu, Yusuke Kobayashi, Christopher M. Heaphy, Alan K. Meeker, Tian-Li Wang, and Ie-Ming Shih. Inactivating ARID1A tumor suppressor enhances TERT transcription and maintains telomere length in cancer cells. Journal of Biological Chemistry, 291(18):9690-9699, 2016.

[40] Marylyn D. Ritchie, Lance W. Hahn, Nady Roodi, L. Renee Bailey, William D. Dupont, Fritz F. Parl, and Jason H. Moore. Multifactor-dimensionality reduction reveals high-order interactions among estrogen-metabolism genes in sporadic breast cancer. American Journal of Human Genetics, 69(1):138-147, July 2001.

[41] Francisco J. R. Ruiz, Isabel Valera, Carlos Blanco, and Fernando Perez-Cruz. Bayesian nonparametric comorbidity analysis of psychiatric disorders. Journal of Machine Learning Research, 15(1):1215-1247, January 2014.

[42] Lori C. Sakoda, Eric Jorgenson, and John S. Witte. Turning of COGS moves forward findings for hormonally mediated cancers. Nature Genetics, 45(4):345-348, April 2013.

[43] Michael R. Stratton, Peter J. Campbell, and P. Andrew Futreal. The cancer genome. Nature, 458(7239):719-724, April 2009.

[44] Yee Whye Teh, Dilan Gorur, and Zoubin Ghahramani. Stick-breaking construction for the Indian buffet process. In International Conference on Artificial Intelligence and Statistics, pp. 556-563, 2007.

[45] Romain Thibaux and Michael I. Jordan. Hierarchical Beta processes and the Indian buffet process. In International Conference on Artificial Intelligence and Statistics, pp. 564-571, 2007.

[46] Zoran Utkovski, Melanie F Pradier, Viktor Stojkoski, Fernando Perez-Cruz, and Ljupco Kocarev. Economic complexity unfolded: Interpretable model for the productive structure of economies. PloS one, 13(8):e0200822, 2018.

[47] Isabel Valera, Melanie F. Pradier, and Zoubin Ghahramani. General latent feature modeling for data exploration tasks. Workshop on Human Iinterpretability in Machine Learning at Neural Information Processing Systems, 2017.

[48] Xiaoyue Wang, Audrey Q. Fu, Megan E. McNerney, and Kevin P. White. Widespread genetic epistasis among cancer genes. Nature Communications, 5:4828, November 2014.

[49] Zhuoran Wang, Anoop D. Shah, A. Rosemary Tate, Spiros Denaxas, John Shawe-Taylor, and Harry Hemingway. Extracting diagnoses and investigation results from unstructured text in electronic health records by semi-supervised machine learning. PLoS ONE, 7(1):e30412, January 2012.

[50] Larry Wasserman. All of statistics: A concise course in statistical inference. Springer Science \& Business Media, 2013.

[51] Ahmet Zehir, Ryma Benayed, Ronak H Shah, Aijazuddin Syed, Sumit Middha, Hyunjae R Kim, Preethi Srinivasan, Jianjiong Gao, Debyani Chakravarty, Sean M Devlin, et al. Mutational landscape of metastatic cancer revealed from prospective clinical sequencing of 10,000 patients. Nature medicine, 23(6):703, 2017.

[52] Wei Zhang, Jun Zhu, Eric E. Schadt, and Jun S. Liu. A Bayesian partition method for detecting pleiotropic and epistatic eQTL modules. PLoS Computational Biology, 6(1), January 2010.

[53] Yu Zhang and Jun S Liu. Bayesian inference of epistatic interactions in case-control studies. Nature Genetics, 39(9):1167-1173, September 2007. 


\section{Appendix: Complete List of Associations}

\subsection{Case-control setup (CC)}

\begin{tabular}{|c|c|c|c|}
\hline Clinical term & Gene & $\beta_{q g}$ & $p$-value \\
\hline FOLFOX Regimen & $\mathrm{APC}$ & 0.66 & $5.12 \mathrm{e}-113$ \\
\hline Leucovorin & $\mathrm{APC}$ & 0.65 & $6.61 \mathrm{e}-65$ \\
\hline Hepatectomy & APC & 0.65 & $1.21 \mathrm{e}-52$ \\
\hline irinotecan & $\mathrm{APC}$ & 0.62 & $4.39 \mathrm{e}-47$ \\
\hline pump (device) & $\mathrm{APC}$ & 0.61 & $2.78 \mathrm{e}-53$ \\
\hline Colorectal Carcinoma & $\mathrm{APC}$ & 0.61 & $2.34 \mathrm{e}-63$ \\
\hline Colorectal & $\mathrm{APC}$ & 0.60 & $2.31 \mathrm{e}-73$ \\
\hline Folinic Acid-Fluorouracil-Irinotecan Regimen & $\mathrm{APC}$ & 0.59 & $1.08 \mathrm{e}-60$ \\
\hline Malignant neoplasm of urinary bladder & TERT & 0.55 & $1.07 \mathrm{e}-61$ \\
\hline S3 (sacral segmental innervation) & APC & 0.54 & $3.58 \mathrm{e}-35$ \\
\hline intrahepatic & $\mathrm{APC}$ & 0.53 & $1.03 \mathrm{e}-30$ \\
\hline Tract & TERT & 0.50 & $1.62 \mathrm{e}-42$ \\
\hline Sigmoid colon & $\mathrm{APC}$ & 0.48 & $6.82 \mathrm{e}-38$ \\
\hline Gross hematuria & TERT & 0.47 & $1.23 \mathrm{e}-47$ \\
\hline Flowcharts (Computer) & $\mathrm{APC}$ & 0.47 & $4.29 \mathrm{e}-32$ \\
\hline Entire intercostal space & $\mathrm{APC}$ & 0.42 & $1.33 \mathrm{e}-41$ \\
\hline Unresectable & $\mathrm{APC}$ & 0.42 & $1.78 \mathrm{e}-20$ \\
\hline Structure of long bone & $\mathrm{APC}$ & 0.42 & $5.86 \mathrm{e}-29$ \\
\hline Midclavicular line & $\mathrm{APC}$ & 0.41 & $1.46 \mathrm{e}-41$ \\
\hline KRAS gene & $\mathrm{APC}$ & 0.39 & $6.40 \mathrm{e}-29$ \\
\hline Cold intolerance & $\mathrm{APC}$ & 0.39 & $1.70 \mathrm{e}-22$ \\
\hline Rectum & $\mathrm{APC}$ & 0.38 & $3.88 \mathrm{e}-26$ \\
\hline Data Port & $\mathrm{APC}$ & 0.37 & $1.35 \mathrm{e}-19$ \\
\hline pump (device) & TP53 & 0.35 & $8.51 \mathrm{e}-17$ \\
\hline Depression motion & $\mathrm{APC}$ & 0.35 & $5.62 \mathrm{e}-22$ \\
\hline Avastin & APC & 0.35 & $1.58 \mathrm{e}-22$ \\
\hline KRAS gene & KRAS & 0.33 & $2.44 \mathrm{e}-23$ \\
\hline FOLFOX Regimen & KRAS & 0.32 & $1.96 \mathrm{e}-32$ \\
\hline Stage IV Lung Adenocarcinoma & EGFR & 0.32 & $2.07 \mathrm{e}-29$ \\
\hline Tract & KDM6A & 0.31 & $1.66 \mathrm{e}-24$ \\
\hline Unresectable & TP53 & 0.31 & $3.62 \mathrm{e}-10$ \\
\hline capecitabine & $\mathrm{APC}$ & 0.30 & $1.94 \mathrm{e}-15$ \\
\hline Imodium & $\mathrm{APC}$ & 0.30 & $6.51 \mathrm{e}-15$ \\
\hline Ulcer & $\mathrm{APC}$ & 0.30 & $3.96 \mathrm{e}-12$ \\
\hline Flushing & $\mathrm{APC}$ & 0.30 & $1.54 \mathrm{e}-11$ \\
\hline Folinic Acid-Fluorouracil-Irinotecan Regimen & KRAS & 0.30 & $1.16 \mathrm{e}-18$ \\
\hline irinotecan & KRAS & 0.30 & $5.60 \mathrm{e}-14$ \\
\hline FOLFOX Regimen & TP53 & 0.29 & $4.61 \mathrm{e}-19$ \\
\hline Hepatectomy & TP53 & 0.29 & $1.19 \mathrm{e}-10$ \\
\hline S3 (sacral segmental innervation) & TP53 & 0.29 & $1.02 \mathrm{e}-09$ \\
\hline Colorectal & KRAS & 0.29 & $2.18 \mathrm{e}-21$ \\
\hline Leucovorin & KRAS & 0.29 & $8.95 \mathrm{e}-16$ \\
\hline Ablation & $\mathrm{APC}$ & 0.29 & $5.61 \mathrm{e}-15$ \\
\hline $\begin{array}{l}\text { Rash and Dermatitis Adverse Event Associated with Chemora- } \\
\text { diation }\end{array}$ & APC & 0.29 & $5.40 \mathrm{e}-13$ \\
\hline Pulse taking & $\mathrm{APC}$ & 0.29 & $2.28 \mathrm{e}-15$ \\
\hline Malignant neoplasm of urinary bladder & KDM6A & 0.29 & $5.14 \mathrm{e}-27$ \\
\hline Potassium Ion & KRAS & 0.28 & $4.17 \mathrm{e}-14$ \\
\hline Renal function & TERT & 0.28 & $8.40 \mathrm{e}-12$ \\
\hline Structure of long bone & TP53 & 0.27 & $5.79 \mathrm{e}-11$ \\
\hline Hydronephrosis & TERT & 0.27 & $3.21 \mathrm{e}-12$ \\
\hline Hepatectomy & KRAS & 0.27 & $7.27 \mathrm{e}-12$ \\
\hline Xeloda & $\mathrm{APC}$ & 0.26 & $1.53 \mathrm{e}-11$ \\
\hline Midclavicular line & TP53 & 0.26 & $6.98 \mathrm{e}-15$ \\
\hline Adenocarcinoma of lung (disorder) & EGFR & 0.26 & $1.49 \mathrm{e}-22$ \\
\hline Leucovorin & TP53 & 0.26 & $1.57 \mathrm{e}-09$ \\
\hline Entire intercostal space & TP53 & 0.26 & $1.47 \mathrm{e}-13$ \\
\hline
\end{tabular}


bioRxiv preprint doi: https://doi.org/10.1101/623215; this version posted April 30, 2019. The copyright holder for this preprint (which was not

Data Port

Gross hematuria

Folinic Acid-Fluorouracil-Irinotecan Regimen

Colorectal Carcinoma

pump (device)

Sigmoid colon

Potassium Ion

Colorectal Carcinoma

Immunotherapy

intrahepatic

Rectum

Neutrophil count decreased

Response process

Tract

Colorectal

Flowcharts (Computer)

Tract

Sigmoid colon

Flowcharts (Computer)

Rectal hemorrhage

Avastin

Bilateral Salpingectomy with Oophorectomy

Unresectable

Urology

Depression motion

irinotecan

Combined Modality Therapy

hearing impairment

Malignant neoplasm of urinary bladder

Gross hematuria

Adriamycin

pemetrexed

Entire intercostal space

S3 (sacral segmental innervation)

Non-Small Cell Lung Carcinoma

Rash and Dermatitis Adverse Event Associated with Chemoradiation

Creatinine

Bone Mineral Density Test

Hydronephrosis

Cold intolerance

intrahepatic

Avastin

KRAS gene

Lobectomy

Malignant neoplasm of urinary bladder

lung lesion

Attribution

Simple mastectomy

Renal function

Pleura

Gross hematuria

Pulmonary function tests

Tract

Invasive Ductal Breast Carcinoma

Midclavicular line

capecitabine

Lumpectomy of breast

Malignant neoplasm of urinary bladder

Incontinence

chain of objects

Renal function

Stent, device

Superficial

Tibialis anterior muscle structure

\begin{tabular}{|c|c|c|}
\hline KRAS & 0.26 & $4.25 \mathrm{e}-11$ \\
\hline KDM6A & 0.26 & $4.71 \mathrm{e}-22$ \\
\hline TP53 & 0.26 & $8.08 \mathrm{e}-11$ \\
\hline TP53 & 0.24 & $1.39 \mathrm{e}-09$ \\
\hline KRAS & 0.24 & $2.59 \mathrm{e}-11$ \\
\hline ТP53 & 0.24 & $3.38 \mathrm{e}-09$ \\
\hline $\mathrm{APC}$ & 0.24 & $4.02 \mathrm{e}-10$ \\
\hline KRAS & 0.23 & $3.18 \mathrm{e}-12$ \\
\hline TERT & 0.23 & $2.76 \mathrm{e}-12$ \\
\hline TP53 & 0.23 & $1.37 \mathrm{e}-05$ \\
\hline TP53 & 0.22 & $5.43 \mathrm{e}-08$ \\
\hline APC & 0.22 & $8.18 \mathrm{e}-07$ \\
\hline $\mathrm{APC}$ & 0.22 & $9.43 \mathrm{e}-07$ \\
\hline FGFR3 & 0.22 & $1.16 \mathrm{e}-15$ \\
\hline TP53 & 0.21 & $5.78 \mathrm{e}-09$ \\
\hline TP53 & 0.21 & $1.46 \mathrm{e}-06$ \\
\hline ARID1A & 0.21 & $5.61 \mathrm{e}-12$ \\
\hline KRAS & 0.21 & $6.84 \mathrm{e}-10$ \\
\hline KRAS & 0.21 & $1.20 \mathrm{e}-08$ \\
\hline APC & 0.21 & $1.88 \mathrm{e}-09$ \\
\hline TP53 & 0.21 & $4.41 \mathrm{e}-07$ \\
\hline PIK3CA & 0.20 & $1.65 \mathrm{e}-06$ \\
\hline KRAS & 0.20 & $6.65 \mathrm{e}-07$ \\
\hline TERT & 0.20 & $8.42 \mathrm{e}-09$ \\
\hline TP53 & 0.20 & $1.43 \mathrm{e}-06$ \\
\hline TP53 & 0.20 & $3.11 \mathrm{e}-05$ \\
\hline $\mathrm{APC}$ & 0.20 & $4.41 \mathrm{e}-06$ \\
\hline TERT & 0.19 & $1.41 \mathrm{e}-06$ \\
\hline FGFR3 & 0.19 & $4.27 \mathrm{e}-15$ \\
\hline FGFR3 & 0.18 & $3.11 \mathrm{e}-14$ \\
\hline PIK3CA & 0.18 & $4.47 \mathrm{e}-06$ \\
\hline EGFR & 0.18 & $2.06 \mathrm{e}-12$ \\
\hline KRAS & 0.18 & $2.80 \mathrm{e}-10$ \\
\hline KRAS & 0.18 & $1.25 \mathrm{e}-05$ \\
\hline EGFR & 0.18 & $4.11 \mathrm{e}-11$ \\
\hline KRAS & 0.18 & $6.24 \mathrm{e}-06$ \\
\hline TERT & 0.17 & $1.24 \mathrm{e}-05$ \\
\hline PIK3CA & 0.17 & $1.65 \mathrm{e}-06$ \\
\hline KDM6A & 0.17 & $1.01 \mathrm{e}-07$ \\
\hline KRAS & 0.17 & $2.64 \mathrm{e}-06$ \\
\hline KRAS & 0.17 & $3.49 \mathrm{e}-05$ \\
\hline KRAS & 0.17 & $3.87 \mathrm{e}-07$ \\
\hline TP53 & 0.17 & $1.28 \mathrm{e}-05$ \\
\hline EGFR & 0.16 & $1.72 \mathrm{e}-08$ \\
\hline ARID1A & 0.16 & $3.25 \mathrm{e}-09$ \\
\hline APC & 0.16 & $8.22 \mathrm{e}-06$ \\
\hline EGFR & 0.16 & $8.10 \mathrm{e}-06$ \\
\hline PIK3CA & 0.16 & $3.40 \mathrm{e}-08$ \\
\hline ARID1A & 0.16 & $8.41 \mathrm{e}-06$ \\
\hline EGFR & 0.16 & $4.16 \mathrm{e}-07$ \\
\hline ARID1A & 0.16 & $1.34 \mathrm{e}-08$ \\
\hline STK11 & 0.16 & $2.19 \mathrm{e}-09$ \\
\hline CREBBP & 0.16 & $8.83 \mathrm{e}-10$ \\
\hline PIK3CA & 0.15 & $8.87 \mathrm{e}-09$ \\
\hline KRAS & 0.15 & $5.38 \mathrm{e}-08$ \\
\hline KRAS & 0.15 & $1.89 \mathrm{e}-05$ \\
\hline PIK3CA & 0.15 & $7.20 \mathrm{e}-07$ \\
\hline ERBB2 & 0.15 & $1.40 \mathrm{e}-10$ \\
\hline TERT & 0.15 & $1.07 \mathrm{e}-06$ \\
\hline TERT & 0.15 & $1.08 \mathrm{e}-05$ \\
\hline KDM6A & 0.15 & $6.54 \mathrm{e}-06$ \\
\hline TERT & 0.14 & $4.23 \mathrm{e}-06$ \\
\hline TERT & 0.14 & $1.68 \mathrm{e}-05$ \\
\hline EGFR & 0.14 & $1.34 \mathrm{e}-06$ \\
\hline
\end{tabular}


bioRxiv preprint doi: https://doi.org/10.1101/623215; this version posted April 30, 2019. The copyright holder for this preprint (which was not aCC-BY 4.0 International license.

Thoracotomy

Tract

Urology

Lobectomy

Malignant neoplasm of urinary bladder

Lobectomy

Tract

Malignant neoplasm of urinary bladder

Leucovorin

Tract

Tract

Bilateral Salpingectomy with Oophorectomy

Non-Small Cell Lung Carcinoma

Non-Small Cell Lung Carcinoma

Folinic Acid-Fluorouracil-Irinotecan Regimen

Malignant neoplasm of urinary bladder

Pulmonary function tests

Colorectal Carcinoma

FOLFOX Regimen

Tract

Urology

KRAS gene

irinotecan

Immunotherapy

Rash and Dermatitis Adverse Event Associated with Chemora-

diation

Tract

Superficial

Immunotherapy

Colorectal

Malignant neoplasm of urinary bladder

Non-Small Cell Lung Carcinoma

Adenocarcinoma of lung (disorder)

Tract

Gross hematuria

Gross hematuria

Tract

Gross hematuria

Sigmoid colon

Tract

Non-Small Cell Lung Carcinoma

Invasive Ductal Breast Carcinoma

Malignant neoplasm of urinary bladder

Sigmoid colon

Colorectal

Adenocarcinoma of lung (disorder)

Simple mastectomy

pemetrexed

Extracapsular

Malignant neoplasm of urinary bladder

Malignant neoplasm of urinary bladder pemetrexed

Colorectal

Malignant neoplasm of urinary bladder

Colorectal

Stage IV Lung Adenocarcinoma

FOLFOX Regimen

Non-Small Cell Lung Carcinoma

Prostate carcinoma

\begin{tabular}{|c|c|c|}
\hline STK11 & 0.14 & $9.65 \mathrm{e}-07$ \\
\hline ERBB2 & 0.14 & $1.87 \mathrm{e}-07$ \\
\hline KDM6A & 0.13 & $2.55 \mathrm{e}-06$ \\
\hline STK11 & 0.13 & $1.25 \mathrm{e}-07$ \\
\hline RB1 & 0.13 & $5.74 \mathrm{e}-08$ \\
\hline KEAP1 & 0.13 & $1.22 \mathrm{e}-08$ \\
\hline STAG2 & 0.13 & $4.54 \mathrm{e}-07$ \\
\hline CREBBP & 0.12 & $8.84 \mathrm{e}-08$ \\
\hline SMAD4 & 0.12 & $5.31 \mathrm{e}-08$ \\
\hline PBRM1 & 0.12 & $2.11 \mathrm{e}-07$ \\
\hline ROS1 & 0.12 & $1.89 \mathrm{e}-05$ \\
\hline GATA3 & 0.12 & $1.93 \mathrm{e}-05$ \\
\hline STK11 & 0.12 & $1.09 \mathrm{e}-07$ \\
\hline KRAS & 0.12 & $8.61 \mathrm{e}-05$ \\
\hline SMAD4 & 0.11 & $8.21 \mathrm{e}-08$ \\
\hline EP300 & 0.11 & $2.57 \mathrm{e}-07$ \\
\hline KEAP1 & 0.11 & $8.29 \mathrm{e}-07$ \\
\hline SMAD4 & 0.11 & $4.47 \mathrm{e}-07$ \\
\hline SMAD4 & 0.11 & $2.03 \mathrm{e}-10$ \\
\hline FAT1 & 0.11 & $3.77 \mathrm{e}-05$ \\
\hline ERBB2 & 0.11 & $1.95 \mathrm{e}-05$ \\
\hline SMAD4 & 0.11 & $4.34 \mathrm{e}-07$ \\
\hline SMAD4 & 0.11 & $1.45 \mathrm{e}-05$ \\
\hline RBM10 & 0.11 & $9.55 \mathrm{e}-06$ \\
\hline TCF7L2 & 0.11 & $2.14 \mathrm{e}-05$ \\
\hline EP300 & 0.10 & $1.24 \mathrm{e}-05$ \\
\hline FGFR3 & 0.10 & $2.29 \mathrm{e}-05$ \\
\hline FGFR3 & 0.10 & $4.13 \mathrm{e}-05$ \\
\hline PTPRS & 0.10 & $9.11 \mathrm{e}-07$ \\
\hline ATM & 0.10 & $8.03 \mathrm{e}-05$ \\
\hline PTPRD & 0.10 & $9.64 \mathrm{e}-06$ \\
\hline KEAP1 & 0.10 & $1.46 \mathrm{e}-07$ \\
\hline SPEN & 0.10 & $3.32 \mathrm{e}-05$ \\
\hline CREBBP & 0.10 & $1.43 \mathrm{e}-05$ \\
\hline NSD1 & 0.10 & $7.95 \mathrm{e}-07$ \\
\hline FANCA & 0.10 & $3.20 \mathrm{e}-05$ \\
\hline ERBB2 & 0.10 & $1.81 \mathrm{e}-05$ \\
\hline TCF7L2 & 0.10 & $6.46 \mathrm{e}-06$ \\
\hline ERBB3 & 0.10 & $2.09 \mathrm{e}-05$ \\
\hline KEAP1 & 0.10 & $1.02 \mathrm{e}-06$ \\
\hline GATA3 & 0.10 & $3.71 \mathrm{e}-08$ \\
\hline ERBB3 & 0.09 & $6.40 \mathrm{e}-06$ \\
\hline SMAD4 & 0.09 & $3.46 \mathrm{e}-05$ \\
\hline ERBB4 & 0.09 & $3.22 \mathrm{e}-05$ \\
\hline STK11 & 0.09 & $1.91 \mathrm{e}-05$ \\
\hline GATA3 & 0.09 & $2.59 \mathrm{e}-06$ \\
\hline STK11 & 0.09 & $1.23 \mathrm{e}-05$ \\
\hline FOXA1 & 0.08 & $3.92 \mathrm{e}-05$ \\
\hline PBRM1 & 0.08 & $7.37 \mathrm{e}-05$ \\
\hline NSD1 & 0.08 & $4.75 \mathrm{e}-05$ \\
\hline KEAP1 & 0.08 & $1.31 \mathrm{e}-05$ \\
\hline SMAD4 & 0.08 & $1.87 \mathrm{e}-05$ \\
\hline BRCA1 & 0.08 & $1.80 \mathrm{e}-04$ \\
\hline TCF7L2 & 0.08 & $2.29 \mathrm{e}-05$ \\
\hline RBM10 & 0.08 & $5.16 \mathrm{e}-05$ \\
\hline PTPRS & 0.08 & $1.31 \mathrm{e}-05$ \\
\hline EPHA3 & 0.08 & $7.61 \mathrm{e}-05$ \\
\hline FOXA1 & 0.07 & $3.61 \mathrm{e}-05$ \\
\hline
\end{tabular}

Table 6: Complete list of clinico-genetic associations found using the Case-Control Set-up. $\beta_{q g}$ refers to the linear weight as described in Section 2.4. Associations in bold have also been discovered by the H-PFA. 


\subsection{Hierarchical Poisson Factor Analysis}

$100 \%$ patients with prostate cancer

\begin{tabular}{|c|c|c|c|c|c|}
\hline \multicolumn{3}{|c|}{ Clinical record } & \multicolumn{3}{|c|}{ Genetic information } \\
\hline Phenotype $q$ & $A_{k q}$ & $p$-value & Gene $g$ & $A_{k g}$ & $p$-value \\
\hline Prostate carcinoma & 0.63 & $4.06 \mathrm{e}-43$ & None & & \\
\hline Biopsy of prostate & 0.52 & $1.63 \mathrm{e}-34$ & & & \\
\hline Extracapsular & 0.48 & $3.54 \mathrm{e}-32$ & & & \\
\hline adenocarcinoma of the prostate & 0.43 & $3.98 \mathrm{e}-26$ & & & \\
\hline Personal Attribute & 0.33 & $2.23 \mathrm{e}-16$ & & & \\
\hline Robotics & 0.25 & $5.86 \mathrm{e}-13$ & & & \\
\hline Pelvic lymph node group & 0.20 & $2.34 \mathrm{e}-09$ & & & \\
\hline Lupron & 0.20 & $2.37 \mathrm{e}-07$ & & & \\
\hline Incontinence & 0.17 & $7.87 \mathrm{e}-07$ & & & \\
\hline External Beam Radiation Therapy & 0.17 & $1.04 \mathrm{e}-06$ & & & \\
\hline Positive Surgical Margin & 0.14 & $7.71 \mathrm{e}-06$ & & & \\
\hline
\end{tabular}

$32.3 \%$ patients with breast carcinoma, $41.2 \%$ non-small cell lung cancer, $26.5 \%$ prostate cancer

\begin{tabular}{|c|c|c|c|c|c|}
\hline \multicolumn{3}{|c|}{ Clinical record } & \multicolumn{3}{|c|}{ Genetic information } \\
\hline Phenotype $q$ & $A_{k q}$ & $p$-value & Gene $g$ & $A_{k g}$ & $p$-value \\
\hline Invasive Ductal Breast Carcinoma & 0.47 & $1.86 \mathrm{e}-11$ & None & & \\
\hline Adriamycin & 0.37 & $6.54 \mathrm{e}-11$ & & & \\
\hline Lytic lesion & 0.33 & $2.45 \mathrm{e}-09$ & & & \\
\hline Lumpectomy of breast & 0.33 & $4.86 \mathrm{e}-08$ & & & \\
\hline Zometa & 0.32 & $2.47 \mathrm{e}-08$ & & & \\
\hline Palliative Care & 0.22 & $8.32 \mathrm{e}-06$ & & & \\
\hline
\end{tabular}

Table 7: Additional clinical associations (complex phenotypes) found by the H-PFA.

\begin{tabular}{|c|c|c|c|c|c|}
\hline \multicolumn{3}{|l|}{ Clinical record } & \multicolumn{3}{|c|}{ Genetic information } \\
\hline Phenotype $q$ & $A_{k q}$ & $p$-value & Gene $g$ & $A_{k g}$ & $p$-value \\
\hline FOLFOX Regimen & 0.77 & $3.47 \mathrm{e}-19$ & $\mathrm{APC}$ & 0.59 & $5.34 \mathrm{e}-11$ \\
\hline Rectum & 0.59 & $1.56 \mathrm{e}-14$ & & & \\
\hline Sigmoid colon & 0.43 & $8.36 \mathrm{e}-09$ & & & \\
\hline $\begin{array}{l}\text { Rash and Dermatitis Adverse Event Associated } \\
\text { with Chemoradiation }\end{array}$ & 0.42 & $1.24 \mathrm{e}-09$ & & & \\
\hline capecitabine & 0.42 & $5.37 \mathrm{e}-09$ & & & \\
\hline Colorectal & 0.42 & $1.19 \mathrm{e}-07$ & & & \\
\hline Folinic Acid-Fluorouracil-Irinotecan Regimen & 0.40 & $1.11 \mathrm{e}-07$ & & & \\
\hline KRAS gene & 0.38 & $2.12 \mathrm{e}-07$ & & & \\
\hline Ulcer & 0.36 & $3.64 \mathrm{e}-08$ & & & \\
\hline irinotecan & 0.35 & $3.10 \mathrm{e}-07$ & & & \\
\hline Rectal hemorrhage & 0.34 & $3.31 \mathrm{e}-06$ & & & \\
\hline Avastin & 0.32 & $1.13 \mathrm{e}-05$ & & & \\
\hline Leucovorin & 0.29 & $2.95 \mathrm{e}-05$ & & & \\
\hline Combined Modality Therapy & 0.26 & $5.02 \mathrm{e}-05$ & & & \\
\hline Response to treatment & 0.25 & $4.10 \mathrm{e}-05$ & & & \\
\hline
\end{tabular}

Table 8: Additional clinico-genetic association found by the H-PFA involving APC gene. This group of associations was found in a subgroup of $100 \%$ bladder cancer patients. 


\begin{tabular}{llllll}
\hline \multicolumn{2}{c}{ Clinical record } & & \multicolumn{2}{c}{ Genetic information } \\
\hline \hline Phenotype $q$ & $A_{k q}$ & $p$-value & Gene $g$ & $A_{k g}$ & $p$-value \\
\hline Stage IV Lung Adenocarcinoma & 0.64 & $3.47 \mathrm{e}-15$ & EGFR & 0.35 & $8.65 \mathrm{e}-06$ \\
Adenocarcinoma of lung (disorder) & 0.56 & $3.92 \mathrm{e}-11$ & & & \\
pemetrexed & 0.51 & $7.89 \mathrm{e}-10$ & & & \\
Tibialis anterior muscle structure & 0.32 & $3.40 \mathrm{e}-06$ & & & \\
\hline
\end{tabular}

Table 9: Additional clinico-genetic associations found by the H-PFA involving EGFR gene. This group of associations was found in a subgroup of $100 \%$ non-small cell lung cancer patients.

\section{Appendix: Inference details for Poisson Factor Analysis}

Poisson factorization models have been successfully applied for recommendation systems [15], topic modeling [14], and analysis of Electronic Health Records among others [21]. Let $\mathbf{X} \in \mathbb{N}^{N \times D}$ be a sparse matrix of count-data observations with $N$ samples and $D$ dimensions. The generative model for the Bernoulli process Poisson factor analysis (PFA) is given by:

$$
\begin{aligned}
x_{n d} & \sim \operatorname{Poisson}\left(\mathbf{Z}_{n \bullet} \mathbf{A} \bullet d\right) \\
\mathbf{Z} & \sim \operatorname{IBP}(\alpha) \\
\mathbf{A}_{k d} & \sim \operatorname{Gamma}\left(\alpha_{A}, \frac{\mu_{A}}{\alpha_{A}}\right),
\end{aligned}
$$

where $\mathbf{Z}$ is a $N \times K$ matrix of binary weights, and $\mathbf{A}$ is a $K \times D$ matrix of non-negative hidden factors. Direct inference in such models is intractable, but we can easily solve the problem using MCMC techniques. For each observation $x_{n d}$, we introduce the auxiliary variables $x_{n d, 1}^{\prime}, \ldots, x_{n d, K}^{\prime}$ such that $x_{n d}=\sum_{k=1}^{K} x_{n d, k}^{\prime}$, and $x_{n d, k}^{\prime} \sim \operatorname{Poisson}\left(\theta_{n k} A_{k d}\right)$ for $k=1, \ldots, K$. Each Poisson count is separated in a sum of Poisson contributions corresponding to each latent factor. Given such auxiliary variables, the model is conditionally conjugate, and a Gibbs sampler can be derived straightforwardly. In particular, we use the following theorem:

Theorem 1 Let $Y_{1}, \ldots, Y_{n}$ be Poisson distributed random variables with rates $\lambda_{1}, \ldots, \lambda_{n}$ respectively. Let us define $S=\sum_{n=1}^{N} Y_{n}$. Then,

$$
\left\{Y_{1}, \ldots, Y_{n}\right\} \mid S \sim \operatorname{Multinomial}\left(\left\{\frac{\lambda_{i}}{\sum_{n=1}^{N} \lambda_{n}}\right\}_{i}, S\right) .
$$

Using Theorem $1, \boldsymbol{x}_{\boldsymbol{n} \boldsymbol{d}, \boldsymbol{\bullet}}^{\prime}$ can be sampled from a Multinomial given $x_{n d}, \boldsymbol{\theta}_{\boldsymbol{n} \bullet}$ and $\mathbf{A} \bullet d$. In the following, we propose two MCMC algorithms: a collapsed Gibbs sampler where matrix $\mathbf{A}$ is marginalized out using a Laplace approximation, and an uncollapsed slice sampler version which allows for parallel sampling of both the elements in $\mathbf{Z}$ and $\mathbf{A}$ given the auxiliary variables $x_{n d, 1}^{\prime}, \ldots, x_{n d, K}^{\prime}$. The results shown in this work have been generated using the uncollapsed Gibbs Sampler.

\subsection{Collapsed Gibbs Sampler}

We first propose a collapsed Gibbs sampler where matrix $\mathbf{A}$ is marginalized out, and we only need to sample the elements of matrix $\mathbf{Z}$. We need to compute its posterior distribution:

$$
\begin{aligned}
p\left(z_{n k} \mid \mathbf{X}, \mathbf{Z}_{\neg n k}\right) & \propto p\left(z_{n k} \mid \mathbf{Z}_{\neg n k}\right) p(\mathbf{X} \mid \mathbf{Z}) \\
& \propto p\left(z_{n k} \mid \mathbf{Z}_{\neg n k}\right) \int p(\mathbf{X} \mid \mathbf{Z}, \mathbf{A}) p(\mathbf{A}) d \mathbf{A} \\
& \propto p\left(z_{n k} \mid \mathbf{Z}_{\neg n k}\right) \prod_{d=1}^{D} \int\left(\prod_{n=1}^{N} p\left(x_{n d} \mid \mathbf{Z}_{n \bullet}, \mathbf{A}_{\bullet d}\right)\right) p\left(\mathbf{A}_{\bullet}\right) d \mathbf{A}_{\bullet} d
\end{aligned}
$$


In order to approximate the integral in (16), we resort to a Laplace approximation, which assumes that:

$$
\int e^{\psi(\mathbf{A} \bullet d)} d \mathbf{A} \bullet d
$$

has a peak at a certain value of $\mathbf{A}_{\bullet}^{\mathrm{MAP}}$. The idea is to Taylor-expand the un-normalized logposterior of $\mathbf{A}_{\bullet} d$ and approximate $e^{\psi\left(\mathbf{A}_{\bullet}\right)}$ by an unnormalized Gaussian. The integral thus corresponds to the normalizing constant of this Gaussian, in our case:

$$
\int\left(\prod_{n=1}^{N} p\left(x_{n d} \mid \mathbf{Z}_{n \bullet}, \mathbf{A}_{\bullet}\right)\right) p\left(\mathbf{A}_{\bullet}\right) d \mathbf{A}_{\bullet} d=e^{\psi\left(\mathbf{A}_{\bullet d}^{\mathrm{MAP}}\right)} \sqrt{\frac{(2 \pi)^{K}}{\left|-\nabla \nabla \psi\left(\mathbf{A}_{\bullet d}^{\mathrm{MAP}}\right)\right|}}
$$

Equations to find maximum a posteriori $\mathbf{A}_{\bullet d}^{\mathrm{MAP}}$. Let us define $\psi\left(\mathbf{A}_{\bullet}\right)$ as the unnormalized log-posterior of $\mathbf{A}_{\bullet}$, i.e,

$$
\begin{aligned}
& \psi(\mathbf{A} \bullet d)=\sum_{n=1}^{N} \log p\left(x_{n d} \mid \mathbf{Z}_{n \bullet}, \mathbf{A}_{\bullet}\right)+\log p\left(\mathbf{A}_{\bullet}\right) \\
& \psi(\mathbf{A} \bullet d)=\sum_{n=1}^{N} x_{n d} \log \left(\mathbf{Z}_{n \bullet \mathbf{A}} \mathbf{A} d\right)-\sum_{n=1}^{N} \mathbf{Z}_{n} \bullet \mathbf{A}_{\bullet} d+\sum_{k=1}^{K}\left(\alpha_{A}-1\right) \log \mathbf{A}_{k d}-\frac{\alpha_{A}}{\mu_{A}} \sum_{k=1}^{K} \mathbf{A}_{k d}+R \\
& \text { where } R=-\sum_{n=1}^{N} \log x_{n d} !-K\left(\alpha_{A} \log \frac{\mu_{A}}{\alpha_{A}}+\log \Gamma\left(\alpha_{A}\right)\right) \\
& \nabla \psi(\mathbf{A} \bullet d)=\sum_{n=1}^{N} x_{n d} \frac{\mathbf{Z}_{n \bullet}}{\mathbf{Z}_{n \bullet} \cdot \mathbf{A}_{\bullet d}}-\sum^{N} \mathbf{z}_{n \bullet}+\left(\alpha_{A}-1\right) \frac{1}{\mathbf{A} \bullet d}-\frac{\alpha_{A}}{\mu_{A}} \\
& \nabla \nabla \psi(\mathbf{A} \bullet d)=-\sum_{n=1}^{N} x_{n d} \frac{\mathbf{Z}_{n} \bullet \mathbf{Z}_{n \bullet}^{T}}{\left(\mathbf{Z}_{n \bullet} \bullet \mathbf{A}_{\bullet}\right)^{2}}-\left(\frac{\alpha_{A}-1}{\mathbf{A}_{\bullet d}^{2}}\right)^{T} \mathbf{I}
\end{aligned}
$$

In order to find the maximum value $\mathbf{A}_{\bullet d}^{\mathrm{MAP}}$, we can use either Newton's method or gradient descent. Where applicable, Newton's method might converge faster towards a local maximum or minimum than gradient descent. Newton's method is an iterative method for optimization where each value $\mathbf{A}_{\bullet d}^{(t)}$ at iteration $t$ is computed as:

$$
\mathbf{A}_{\bullet d}^{(t)}=\mathbf{A}_{\bullet d}^{(t-1)}+\gamma\left[\nabla \nabla \psi\left(\mathbf{A}_{\bullet d}^{(t-1)}\right)\right]^{-1} \nabla \psi\left(\mathbf{A}_{\bullet d}^{(t-1)}\right)
$$

where $\gamma \in(0,1]$ is the step-size of the algorithm. Note that for the Laplace approximation to work properly, $-\nabla \nabla \psi\left(\mathbf{A}_{\bullet}\right)$ should be a positive semi-definite matrix. This is guaranteed only if $\alpha_{B}>1$, so the collapsed Gibbs sampler will only work for shape parameters bigger than one, resulting in non-sparse $\mathbf{A}$ matrices.

\subsection{Uncollapsed Gibbs Sampler}

Inference for the PFA model can be performed using an uncollapsed Gibbs sampler together with a slice sampler for semi-ordered stick-breaking representation of the IBP [44]. For the sake of completeness, the slice sampling procedure for matrix $\mathbf{Z}$ is described in Algorithm 2. Using the auxiliary random variables described at the beginning of this Appendix, the complete conditionals can be easily derived as follows:

$$
\begin{aligned}
& p\left(A_{k d} \mid \mathbf{Z}_{\bullet k}, \boldsymbol{x}_{\bullet d, \boldsymbol{k}}^{\prime}\right) \propto \operatorname{Gamma}\left(c+\sum_{n=1}^{N} x_{n d, k}^{\prime}, d+\sum_{n=1}^{N} z_{n k}\right) \\
& p\left(\boldsymbol{x}_{\boldsymbol{n} d, \bullet}^{\prime} \mid x_{n d}, \mathbf{A}_{\bullet}\right) \propto \underset{23}{\operatorname{Multinomial}}\left(\left\{\frac{z_{n i} A_{i d}}{\sum_{k=1}^{K} z_{n k} A_{k d}}\right\}_{i=1}^{K}, x_{n d}\right)
\end{aligned}
$$


Algorithm 2 Slice sampler for the semi-ordered stick-breaking representation of the IBP [44].

1: Sample auxiliary slice variable $s$ for the creation of new sticks, if $s<\mu_{\left(K^{\star}\right)}$, create new sticks using adaptive rejection sampling, and sample corresponding feature parameters from prior.

2: Sample $\mathbf{Z}$ matrix. Given the stick weights, each row can be sampled independently and in parallel:

$$
p\left(z_{n k} \mid r e s t\right) \propto \mu_{(k)} \cdot \prod^{D} p\left(x_{n d, k}^{\prime} \mid z_{n k}, \theta_{n k}, \mathbf{A}_{k d}\right) .
$$

3: Remove inactive features.

4: Sample sticks

$$
p\left(\mu_{(k)} \mid r e s t\right) \sim \operatorname{Beta}\left(m_{\bullet, k}, 1+N-m_{\bullet, k}\right),
$$

where $m_{\bullet, k}=\sum_{i=1}^{N} z_{n k}$.

$$
\begin{gathered}
\log p\left(z_{n k}=1 \mid \mathbf{Z}_{\neg n k}, \boldsymbol{A}_{\boldsymbol{k} \bullet}, \pi_{k}\right)=\frac{1}{1+\mathrm{e}^{-u_{n k}}}, \\
\text { where } u_{n k}=\sum_{d=1}^{D} x_{n d} \log \left(\frac{\sum_{j \neq k} z_{n j} A_{j d}+A_{k d}}{\sum_{j \neq k} z_{n j} A_{j d}}\right)-\sum_{d=1}^{D} A_{k d}+\log \frac{\pi_{k}}{1-\pi_{k}} .
\end{gathered}
$$

Inference for the hierarchical Poisson Factor Analysis (H-PFA) is analoguous to Algorithm 2 except step 4, where we sample the per-category feature probability $\pi_{k}^{l}$ for each cancer type $l$ and feature $k$ from a Beta distribution based on counts per cancer type as follows:

$$
p\left(\pi_{(k)}^{l} \mid r e s t\right) \sim \operatorname{Beta}\left(m_{\bullet, k}^{l}, 1+N_{l}-m_{\bullet, k}^{l}\right),
$$

where $N_{l}$ is the number of patients with cancer type $l, m_{\bullet, k}^{l}$ is the number of patients having feature $k$ active and cancer type $l, r_{n}$ is the cancer type indicator for patient $n$, and $m_{\bullet}^{l}, k=$ $\sum_{i=1}^{N} z_{n k} \mathbf{1}\left[r_{n}=l\right]$. 